\title{
Abhandlungen
}

\section{Ein Vorschlag zur Lösung der europäischen Rechtsstaatlichkeitskrise: die Lehre der Diagonalität}

\author{
Csongor István Nagy* \\ ELKH-Forschungszentrum für Sozialwissenschaften, Budapest und \\ Universität von Szeged, Ungarn \\ csongor.nagy@gmail.com
}

Abstract

$\begin{array}{ll}\text { Keywords } & 304\end{array}$

$\begin{array}{lr}\text { I. Einführung } & 304\end{array}$

II. Rechtswissenschaftliche Lösungsversuche 306

III. Die Diagonalität des Rechtsstaatlichkeitsprinzips der EU: Warum ist die diagonale Anwendung erforderlich?

1. Die Diagonalität des Rechtsstaatlichkeitsprinzips: Begriffsbildung

2. Das herrschende Anwendungsbereichsparadigma und das vorgeschlagene (diagonale) Grundwerteparadigma

IV. Mehrebenen-Verfassungssysteme in rechtsvergleichender Perspektive 315

V. Die Lehre von der Diagonalität $\quad 324$

1. Die ontologische Ebene: Warum ist die Diagonalitätslehre erforderlich? 324

2. Die dogmatische Ebene: die unmittelbare Wirkung von Artikel 2 EUV 330

3. Die institutionelle Ebene: Legalisierung und Entpolitisierung 335

VI. Fazit

Summary: A Proposal for a Solution to the European Rule of Law Crisis: the Doctrine of Diagonality

Keywords

* Professor, Doctor iuris (ELTE, Budapest), LLM (CEU, Budapest/New York/Wien), PhD (ELTE), SJD (CEU), Professor und Leiter des Lehrstuhls für Internationales Privatrecht an der Universität Szeged, Forschungsprofessor an dem ELKH-Forschungszentrum für Sozialwissenschaften. Die Forschung wird durch das Projekt EFOP-3.6.2-16-2017-00007 „Die Aspekte von der Entwicklung einer intelligenten, nachhaltigen und inklusiven Gesellschaft: soziale, technologische Innovationsnetze in der Beschäftigung und in der digitalen Wirtschaft" unterstützt. Das Projekt wird von der Europäischen Union finanziert, und vom Europäischen Sozialfonds und Ungarischen Staatshaushalt kofinanziert. Der Autor ist Dr. Katrin Bohne und Dr. Dóra Frey für Hinweise und Bemerkungen sehr dankbar. 


\section{Abstract}

Der Beitrag macht einen Vorschlag zur Lösung des europäischen Rechtsstaatlichkeitsproblems. Er legt dar, dass das EU-Recht der Lehre der Diagonalität folgen und das derzeit herrschende Paradigma des Anwendungsbereichs durch das Paradigma der Grundwerte ersetzt werden sollte. Der Vorschlag des Beitrags bedient sich der Rechtsvergleichungsmodelle von Mehrebenensystemen im Verfassungsrecht, insbesondere der amerikanischen Doktrin der Inkorporation. In einem ersten Schritt führt der Beitrag den neuen Begriff der Diagonalität für die Beschreibung der europäischen Rechtsstaatlichkeitskrise ein, gibt einen Überblick über den (fehlenden) Status der Diagonalität und übt Kritik an dem herrschenden Paradigma des Anwendungsbereichs. Zudem wird das vorgeschlagene (diagonal wirkende) Paradigma der Grundwerte entwickelt. In einem zweiten Schritt stellt der Beitrag die Lösungsversuche der Rechtswissenschaft dar und zeigt auf, warum diese Vorschläge das europäische Rechtsstaatlichkeitsproblem nicht lösen können. In einem dritten Schritt wird erläutert, dass der diagonale Ansatz ein typisches Phänomen der Mehrebenensysteme darstellt und wie die vergleichende Rechtswissenschaft zur Lösung des Rechtsstaatsproblems der EU beitragen kann. Schließlich stellt der Beitrag die Lehre der Diagonalität im Einzelnen vor und erklärt, warum das herrschende Paradigma des Anwendungsbereichs durch das Paradigma der Grundwerte ersetzt werden sollte. Er legt dar, dass Artikel 2 des Vertrags über die Europäische Union (EUV) ein geeigneter Ausgangspunkt für die Anwendung der Lehre von der Diagonalität ist und der Europäische Gerichtshof (EuGH) die europäischen Rechtsstaatlichkeitsanforderungen nach Artikel 2 EUV gegenüber den Mitgliedstaaten durchsetzen sollte.

\section{Keywords}

Rechtsstaatlichkeit - Rechtsstaatlichkeitskrise - konstitutionelle Identitäten - Mehrebenensysteme

\section{Einführung}

Die Krise der Rechtsstaatlichkeit in der EU, die in einigen Mitgliedstaaten zur Schwächung des Konstitutionalismus geführt hat, und die heftige Debatte darüber, ob und wie die Organe der Europäischen Union (EU) eingreifen 
sollten, haben den Schutz der Rechtsstaatlichkeit zu einer der Kernfragen der europäischen Integration gemacht. ${ }^{1}$ Heutzutage ist es allgemein anerkannt, dass die Wahrung der Rechtsstaatlichkeit ${ }^{2}$ in den Mitgliedstaaten eine Schicksalsfrage des Europaprojekts geworden ist, und die europäische Integration früher oder später eine Antwort darauf geben muss. Diese historische Frage kann die nächste Phase der Geschichte Europas zu einer ,immer engeren Union“ eröffnen. In diesem Sinne, können jene mitgliedstaatlichen Regierungen, die sich der EU und ihren Grundwerten laut und energisch widersetzen, erheblich zu der Schaffung einer weiteren wichtigen europäischen Befugnis beitragen. ${ }^{3}$ Die Geschichte der europäischen Integration hat bewiesen, dass die Herausforderungen, welche die Integration nicht umbringen, sie stärker machen.

Der Beitrag hat diese verfassungsrechtliche Frage zum Gegenstand und macht einen Vorschlag zur Lösung des Rechtsstaatlichkeitsproblems. Er legt dar, dass das EU-Recht der Lehre der Diagonalität folgen und das derzeit herrschende Paradigma des Anwendungsbereichs durch das Paradigma der Grundwerte ersetzt werden sollte. Der Vorschlag des Beitrags bedient sich der Rechtsvergleichungsmodelle von Mehrebenensystemen im Verfassungsrecht, insbesondere der amerikanischen Doktrin der Inkorporation.

In einem ersten Schritt stellt der Beitrag die Lösungsversuche der Rechtswissenschaft dar und zeigt auf, warum diese Vorschläge das europäische Rechtsstaatlichkeitsproblem nicht lösen können.

In einem zweiten Schritt führt der Beitrag den neuen Begriff der Diagonalität für die Beschreibung der europäischen Rechtsstaatlichkeitskrise ein, gibt einen Überblick über den (fehlenden) Status der Diagonalität und übt Kritik an dem herrschenden Paradigma des Anwendungsbereichs. $\mathrm{Zu}$ -

1 Siehe Armin von Bogdandy/Matthias Kottmann/Carlino Antpöhler/Johanna Dickschen/ Simon Hentrei/Maja Smrkolj, Reverse Solange - Protecting the Essence of Fundamental Rights Against EU Member States, CML Rev. 49 (2012), 489-519; Armin von Bogdandy/Michael Ioannidis, Das systemische Defizit: Merkmale, Instrumente und Probleme am Beispiel der Rechtsstaatlichkeit und des neuen Rechtsstaatlichkeitsaufsichtsverfahrens, ZaöRV 74 (2014), 283-328 (327-328); Armin von Bogdandy/Carlino Antpöbler/Michael Ioannidis, Protecting EU Values: Reverse Solange and the Rule of Law Framework, in: András Jakab/Dimitry Kochenov (Hrsg.), The Enforcement of EU Law and Values, Oxford: Oxford University Press 2017, 218233 (219-225).

2 In diesem Beitrag umfassen die Begriffe „Rechtsstaatlichkeitsanforderungen“ und „Rechtsstaatlichkeitsprinzip“ alle aus dem europäischen Konstitutionalismus entspringenden und für die Staatsgewalt geltenden verfassungsrechtlichen Anforderungen (z. B. Gewaltenteilung, Unabhängigkeit der Justiz, Schutz der Menschenrechte). Die Ausdrücke „Menschenrechte" und "Grundrechte" werden als Synonyme verwendet.

3 Marie-Pierre Granger, Federalization through Rights in the EU - A Legal Opportunities Approach, in: Csongor István Nagy (Hrsg.), The EU Bill of Rights' Diagonal Application to Member States, Den Haag: Eleven International Publishing 2018, 39-59 (59). 
dem wird das vorgeschlagene (diagonal wirkende) Paradigma der Grundwerte entwickelt.

In einem dritten Schritt wird erläutert, dass der diagonale Ansatz ein typisches Phänomen der Mehrebenensysteme darstellt und wie die vergleichende Rechtswissenschaft zur Lösung des Rechtsstaatsproblems der EU beitragen kann. Obwohl die EU kein Staat ist, ist sie auch keine internationale Organisation im herkömmlichen Sinne. Der eigenartige Charakter der EU-Rechtsordnung impliziert, dass die EU ein Quasi-Bundesstaat ist und das macht den Vergleich mit föderalen Systemen sinnvoll. Man kann in der politikwissenschaftlichen Literatur zahlreiche Analysen finden, die die staatlichen Merkmale der EU aufzeigen ${ }^{4}$ und verschiedene Aspekte der europäischen Integration im transatlantischen Vergleich erörtern. ${ }^{5}$

Schließlich stellt der Beitrag die Lehre der Diagonalität im Einzelnen vor und erklärt, warum das herrschende Paradigma des Anwendungsbereichs durch das Paradigma der Grundwerte ersetzt werden sollte. Er legt dar, dass Artikel 2 EUV ein geeigneter Ausgangspunkt für die Anwendung der Lehre von der Diagonalität ist und der Europäische Gerichtshof die europäischen Rechtsstaatlichkeitsanforderungen nach Artikel 2 EUV gegenüber den Mitgliedstaaten durchsetzen sollte.

\section{Rechtswissenschaftliche Lösungsversuche}

Die rechtswissenschaftliche Literatur enthält zahlreiche Vorschläge zur Stärkung der Rechtsstaatlichkeit in den Mitgliedstaaten. Einige Autoren sprechen für die Ausdehnung des Anwendungsbereichs der Charta der Grundrechte mit der Konsequenz, dass diese auch für innerstaatliche Angelegenhei-

4 Siehe Robert Schütze, From Dual to Cooperative Federalism: The Changing Structure of European Law, Oxford: Oxford university Press 2009, 70. "[T]he American tradition easily classifies the European Union as a Federal Union. The Union has a mixed or compound structure; and in combining international and national elements, it stands on federal 'middle ground'.” Für generelle Theorien über Föderalismus und deren Anwendung auf die EU, siehe Christoph Schönberger, Die Europäische Union als Bund: Zugleich ein Beitrag zur Verabschiedung des Staatenbund-Bundesstaat-Schemas, AöR 129 (2004); Christoph Schönberger, Unionsbürger: Europas föderales Bürgerrecht in vergleichender Sicht, Tübingen: Mohr Siebeck 2005; Olivier Beaud, Théorie de la Fédération, Paris: Presses Universitaires de Frances 2007).

5 Siehe z.B. R. Daniel Kelemen, The Rules of Federalism: Institutions and Regulatory Politics in the EU and Beyond, Cambridge MA: Harvard University Press 2004; Anand Menon/Martin Schain (Hrsg.), Comparative Federalism: the European Union and the United States in Comparative Perspective, Oxford: Oxford University Press 2006; Michael Burgess, Comparative Federalism: Theory and Practice, London: Routledge 2006; Thomas O. Hueglin/ Alan Fenna, Comparative Federalism: a Systematic Inquiry, (2. Aufl., Toronto, Kanada: University of Toronto Press 2015. 
ten gilt. ${ }^{6}$ In Christos Konstantinidis gegen Stadt Altensteig hat Generalanwalt Jacobs dargestellt, dass die EU-Rechtsstaatlichkeit den Rechtsschutz der europäischen Bürger und Unternehmen, die eine wirtschaftliche Tätigkeit in einem anderen Mitgliedstaat ausüben, umfassen sollte. ${ }^{7}$ In der Literatur wird vorgeschlagen, dass die Kommission verschiedene Verstöße gegen die Rechtsstaatlichkeit bündeln könnte, um die Verletzung von Artikel 2 EUV zu beweisen und ein Vertragsverletzungsverfahren einzuleiten („systemisches Vertragsverletzungsverfahren“ / „systemic infringement procedure“). ${ }^{8}$

Es ist ein gemeinsames Charakteristikum der rechtswissenschaftlichen Vorschläge, dass versucht wird, eine Doktrin auszuarbeiten, die zwei (einander widersprechende) Überlegungen miteinander vereinbart. Einerseits ist es offensichtlich, dass das EU-Recht für den wirksamen Schutz der EU-Rechtsstaatlichkeit sorgen muss. Andererseits ist es eine verfassungsrechtliche Grundforderung, dass die EU-Rechtsstaatlichkeit die nationalen Verfassungsidentitäten respektiert. Die vorgenannten Vorschläge versuchen, diese zwei Überlegungen durch die Beschränkung der diagonalen Anwendung in Einklang zu bringen. Dies impliziert, dass eine überzeugende Lösung nur dann gefunden werden kann, wenn die vollständige von der begrenzten Anwendung in einer sinnvollen und vernünftigen Weise abgegrenzt werden kann. Darauf zielt etwa der Vorschlag ab, die Rolle der EU-Rechtsstaatlichkeit in den Mitgliedstaaten als Makroaufsicht und nicht als individuelles Rechtsmittel zu verstehen..$^{9}$ In dieselbe Richtung zielt der Vorschlag, die EURechtsstaatlichkeit in den Mitgliedstaaten nur mit Einschränkungen zur Anwendung zu bringen.

6 Siehe z.B. Nóra Chronowski, Enhancing the Scope of the Charter of Fundamental Rights?, JURA 13 (2014), 13-21; András Jakab, Application of the EU CFR by National Courts in Purely Domestic Cases, in: Jakab/ Kochenov (Fn. 1), 252-262 (255-260).

7 Schlussantrag des Generalanwalts Jacobs im Fall C-168/91 Christos Konstantinidis v. Stadt Altensteig and Landratsamt Calw, ECLI:EU:C:1992:504, Para. 46, "In my opinion, a Community national who goes to another Member State as a worker or self-employed person [...] is entitled not just to pursue his trade or profession and to enjoy the same living and working conditions as nationals of the host State; he is in addition entitled to assume that, wherever he goes to earn his living in the European Community, he will be treated in accordance with a common code of fundamental values, in particular those laid down in the European Convention on Human Rights. In other words, he is entitled to say 'civis europeus sum' and to invoke that status in order to oppose any violation of his fundamental rights."

$8 \mathrm{Kim}$ Lane Scheppele, Enforcing the Basic Principles of EU Law Through Systemic Infringement Actions, in: Carlos Closa/Dimitry Kochenov (Hrsg.), Reinforcing Rule of Law Oversight in the European Union. Cambridge: Cambridge University Press 2016, 105-132.

9 Carlos Closa/Dimitry Kochenov/Joseph H. H. Weiler, Reinforcing Rule of Law Oversight in the European Union, EUI Working Paper RSCAS 2014/25, 4, "The fact that Article 2 TEU as well as other values-relevant instruments to be discussed [...] are not about micro-management and are only aimed at addressing the gravest concerns is evident. Yet, how do we draw the line between the first and the second type of problems outlined?" 
Der berühmteste Vorschlag in dieser Hinsicht ist die „Reverse Solange“Theorie. ${ }^{10}$ Historisch betrachtet ist die Idee, den diagonalen Menschenrechtsschutz mit der europäischen Bürgerschaft zu verbinden und auf Fälle zu beschränken, wo der Rechtsschutz in den Mitgliedstaaten unzureichend ist, zum ersten Mal von Generalanwalt Maduro im Fall Centro Europa aufgeworfen worden. ${ }^{11}$ Im Sinne von "Reverse Solange“ findet die EURechtsstaatlichkeit in den Mitgliedstaaten keine Anwendung, solange das nationale Recht ein ausreichendes Schutzniveau gewährleistet. Das ist das Gegenteil der Solange-Theorie des Bundesverfassungsgerichts, ${ }^{12}$ wonach die Rechtsakte der EU keiner verfassungsrechtlichen Prüfung nach deutschem Recht unterliegen, solange das EU-Recht einen Schutz vorsieht, dessen Niveau dem des deutschen Rechts im Wesentlichen vergleichbar ist. Der Ausgangspunkt dieser Theorie ist der Begriff der Unionsbürgerschaft. Nach der „Reverse Solange“-Theorie sollten die europäischen Rechtsstaatlichkeitsanforderungen gegen die Mitgliedstaaten nur dann angewendet werden, wenn in dem betroffenen Mitgliedstaat ein "Systemfehler" besteht. ${ }^{13}$ Das heißt, dass die europäischen Rechtsstaatlichkeitsanforderungen nur dann zum Tragen kommen, wenn der Rechtsschutz in einem Mitgliedstaat zusammenbricht.

Obwohl eine solche Lösung einen wichtigen Fortschritt und einen wichtigen Beitrag zur Entwicklung des EU-Rechts darstellen würde, kann sie aus verschiedenen Gründen lückenhaft erscheinen, insbesondere dann, wenn der Systemfehler aus einer Reihe von Rechtsverletzungen besteht. ${ }^{14}$ Soweit die Entscheidung über das Vorliegen der Voraussetzungen („Systemfehler") eine umfassende Bewertung erfordert, sind die EU-Gerichte vermutlich kaum in der Lage, diese vorzunehmen. Wenn die Klage auf einer Reihe von Rechtsverletzungen beruht, würde man erwarten, dass der EuGH beurteilt, ob ein Mitgliedstaat wirklich ein Rechtsstaat ist und die Menschenrechte angemessen schützt. Der EuGH würde in diesem Fall nicht das Verhalten, sondern den - rechtsstaatlichen bzw. nicht-rechtsstaatlichen - Charakter des Staates beurteilen. Dies würde das ganze Verfahren stark politisieren. Ferner bietet

10 Siehe von Bogdandy/Kottmann/Antpöhler/Dickschen/Hentrei/Smrkolj (Fn. 1) und von Bogdandy/Antpöbler/Ioannidis (Fn. 1), 219-225.

11 Schlussantrag des Generalanwalts Maduro im Fall C-380/05 Centro Europa, ECLI:EU: C:2007:505, Para. 20-22.

12 BVerfGE 37, 271 - Solange I; BVerfGE 73, 339 - Solange II.

13 von Bogdandy/Antpöhler/Ioannidis (Fn. 1), 219-225.

14 Vgl. Dimitry Kochenov, On Policing Article 2 TEU Compliance - Reverse Solange and Systemic Infringements Analyzed, Polish Yearbook of International Law 33 (2013), 145-170 $(145,159)$, "The main problem, which thousands of EU citizens are facing is not connected to the systemic meta-violations of rights, but to a myriad of most mundane ones, which are not dealt with by national authorities." 
die „Reverse Solange“ den Opfern der konkreten Rechtsverletzungen, die erst in der Kumulierung den Systemfehler ergeben, nur unzureichenden Schutz. Bevor der Zusammenbruch der Rechtsstaatlichkeit festgestellt wird, bleibt den Opfern der Rechtsverletzungen sowohl auf nationaler als auch auf europäischer Ebene der Rechtsschutz versagt. Kommt indes die Schutzfunktion der EU-Rechtsstaatlichkeit zum Tragen, besteht das Risiko, dass die nationalen Verfassungsidentitäten und der Ermessensspielraum der Mitgliedstaaten unnötig beeinträchtigt werden. Schließlich ist der „Solange“-Ansatz hauptsächlich auf die Behebung institutioneller Defizite zugeschnitten (z. B. die EU-Institutionen können die Pflicht zum Menschenrechtsschutz übernehmen, falls nationale Gerichte diese Pflicht nicht erfüllen) und wirft Probleme bezüglich materieller Grundrechtsstandards auf.

\section{Die Diagonalität des Rechtsstaatlichkeitsprinzips der EU: Warum ist die diagonale Anwendung erforderlich?}

Im Folgenden wird der hier verwendete Begriff der Diagonalität des europäischen Rechtsstaatlichkeitsprinzips im Einzelnen erläutert. Zunächst wird der Begriff definiert und die Theorie der diagonalen Anwendung erklärt. Sodann wird der sich aus dem Fehlen der diagonalen Anwendung ergebende Widerspruch im europäischen Verfassungssystem thematisiert und ausgeführt, dass das herrschende Paradigma des Anwendungsbereichs durch das vorgeschlagene diagonal wirkende Paradigma der Grundwerte ersetzt werden sollte.

\section{Die Diagonalität des Rechtsstaatlichkeitsprinzips: Begriffsbil- dung}

Der für die Beschreibung und Lösung des europäischen Rechtsstaatlichkeitsproblems vorgeschlagene Begriff ist die „Diagonalität“. Dieser Begriff bezieht sich auf die Form der Anwendung der europäischen Rechtsstaatsanforderungen auf die Mitgliedstaaten. Sein Gegenstück ist die „horizontale“ Anwendung, welche sich auf die Anwendung der Rechtsstaatsanforderungen in der Unionsrechtsordnung auf die EU-Organe und ihre „nationalen Beauftragten“, d. h. die das EU-Recht durchführenden Mitgliedstaaten, bezieht. In diesem Begriffssystem gilt die Durchsetzung der mitgliedstaatlichen Verfassungsanforderungen gegen die betreffenden Mitgliedstaaten auch als „horizontale“ Anwendung. 
Das EU-Recht enthält ein umfassendes System von rechtsstaatlichen Anforderungen, die für die EU einschließlich der das EURecht durchführenden Mitgliedstaaten gelten („horizontale Anwendung"). Die für die Mitgliedstaaten geltenden Anforderungen sind aber gegenständlich begrenzt („diagonale Anwendung“). Das europäische Rechtsstaatlichkeitsprinzip ist für die Mitgliedstaaten „ausschließlich bei der Durchführung

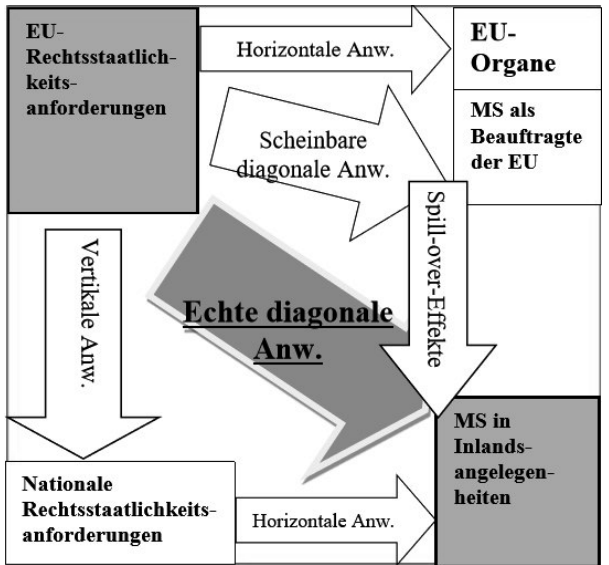
des Rechts der Union" unmittelbar bindend. Obwohl dies erhebliche Spill-Over-Effekte hat, handelt es sich nur um eine scheinbare Diagonalität, da in diesem Fall die Mitgliedstaaten als Beauftragte der EU fungieren. Die echte diagonale Anwendung beschreibt die Fälle, in denen die Mitgliedstaaten in ihren eigenen, nicht durch das Unionsrecht geregelten Angelegenheiten verfahren. Die EU hat einen sehr speziellen Verfassungsaufbau, in dem die politischen und institutionellen Grenzen zwischen der EU und den Mitgliedstaaten voneinander getrennt sind. Die EU verfügt über schwache institutionelle Kapazitäten, daher spielen bei der Durchsetzung des EU-Rechts die mitgliedstaatlichen Behörden und Gerichte eine tragende Rolle. Diese Doppelrolle (Anwendung des EUund nationalen Rechts) impliziert, dass bei der Durchsetzung des EURechts die nationalen Behörden und Gerichte tatsächlich im Namen der EU verfahren.

\section{Das herrschende Anwendungsbereichsparadigma und das vor- geschlagene (diagonale) Grundwerteparadigma}

Derzeit ist die Anwendung des EU-Rechtsstaatlichkeitsprinzips eng mit dem Anwendungsbereich des EU-Rechts (Paradigma des „Anwendungsbereichs") verbunden und der Schwerpunkt der wissenschaftlichen und politischen Rechtsstaatlichkeitsdebatte liegt auf der Bestimmung dieses Anwendungsbereichs. Die Verteidiger des Rechtsstaatlichkeitsprinzips argumentieren, dass der Anwendungsbereich des EU-Rechts weit ausgelegt werden muss, während die betroffenen Mitgliedstaaten für eine enge Auslegung eintreten. Dieser Ansatz der weiten Auslegung des Anwendungsbereichs des 
EU-Rechts greift einerseits zu kurz, weil er in echten nationalen Angelegenheiten keinen Schutz gewährleistet, andererseits zwingt er die EU-Organe, die nach der Wahrung der EU-Grundrechte streben, den Anwendungsbereich des EU-Rechts zu überdehnen.

Die Charta der Grundrechte, die den europäischen Grundrechtekatalog enthält, ist für die EU-Organe bindend, für die Mitgliedstaaten aber nur dann, wenn sie EU-Recht durchführen. ${ }^{15}$ Die Charta bezweckt gar nicht, das Rechtsstaatlichkeitsprinzip gegenüber den Mitgliedstaaten umfassend durchzusetzen. Ganz im Gegenteil, sie bezweckt die Wahrung des Rechtsstaatlichkeitsprinzips im Hinblick auf die EU. Die Idee, dass das EU-Recht die Tätigkeit der EU-Organe und der das EU-Recht anwendenden Mitgliedstaaten europäischen Rechtsstaatlichkeitsanforderungen unterwirft, ergibt sich aus dem Grundsatz, dass ohne verfassungsrechtliche Beschränkungen keine öffentliche Gewalt bestehen darf. ${ }^{16}$ Obwohl der EuGH den Begriff der „Durchführung von EU-Recht“ immer sehr weit auslegt, ${ }^{17}$ hat er diese Unterscheidung nie infrage gestellt. ${ }^{18}$

Es muss betont werden, dass die EU-Organe in dem gegenwärtigen System wegen der Spill-Over-Effekte der „horizontalen“ Anwendung (die in diesem Beitrag als scheinbare Diagonalität bezeichnet wird), zahlreiche Möglichkeiten haben, das Rechtsstaatlichkeitsprinzip gegen die Mitgliedstaaten durchzusetzen. Die Unabhängigkeit der Justiz ist ein sehr gutes Beispiel. ${ }^{19}$ Diese Anforderung ergibt sich nicht aus der diagonalen Anwendung des EURechtsstaatlichkeitsprinzips, sondern aus dem Effizienzgebot des EURechts. Die mitgliedstaatlichen Gerichte haben eine Doppelrolle: Neben dem mitgliedstaatlichen Recht wenden sie auch EU-Recht an. Wegen des Vorabentscheidungsverfahrens haben sie eine entscheidende Rolle in der Durchsetzung des EU-Rechts. Natürliche und juristische Personen können vor

15 Artikel 51. Das ist mit dem Geltungsbereich der allgemeinen Rechtsgrundsätze in Einklang. Siehe Piet Eeckhout, The EU Charter of Fundamental Rights and the Federal Question, CML Rev. 39 (2002), 945-994 (958-969).

16 Siehe Takis Tridimas, The General Principles of EU Law, 1. Aufl., Oxford: Oxford University Press 2006 und Xavier Groussot, General Principles of Community Law, Groningen: Europa Law Publishing 2006; Filippo Fontanelli, The Implementation of European Union Law by Member States under Article 51(1) of the Charter of Fundamental Rights, Columbia Journal of European Law 20 (2014), 194-247, (197-198).

17 EuGH, Urteil v. 26.2.2013, Rs. C-617/10, ECLI:EU:C:2013:105 - Åkerberg Fransson.

18 Siehe EuGH, verb. Rs. C-488/12, 491/12 und C-526/12, ECLI:EU:C:2013:703 - Sándor Nagy u. a.; EuGH, Urteil v. 6.3.2014, Rs. C-206/13, ECLI:EU:C:2014:126 - Siragusa.

19 EuGH, Urteil v. 27.2.2018, Rs. C-64/16, ECLI:EU:C:2018:117 - Associação Sindical dos Juizes Portugueses gegen Tribunal de Contas. Siehe Laurent Pech/Sébastien Platon, A. Court of Justice Judicial independence under threat: The Court of Justice to the Rescue in the ASJP Case, CML Rev. 55 (2018), 1827-1854. EuGH, Urteil v. 24.6.2019, Rs. C-619/18, ECLI:EU: C:2018:910 - Kommission gegen Polen. 
dem EuGH, von einigen beschränkten Ausnahmen abgesehen, keine Klage erheben. Die Fälle aus den Mitgliedstaaten erreichen Luxemburg durch die Vermittlung der mitgliedstaatlichen Gerichte, die im Rahmen des Vorabentscheidungsverfahrens dem EuGH ihre Auslegungsfragen vorlegen. Da diese zwei Rollen nicht institutionell getrennt bzw. voneinander abgegrenzt werden können, impliziert die Pflicht, die Unabhängigkeit der Justiz bezüglich des EU-Rechts sicherzustellen, dass dieselbe Anforderung auch gilt, wenn die Gerichte nationales Recht anwenden. Es ist eine selbstverständliche Erwartung, dass die mitgliedstaatlichen Gerichte bei der Anwendung des EURechts unabhängig sein sollen. Das europäische und das mitgliedstaatliche Recht werden aber von denselben Gerichten angewendet. Deswegen gilt die für die Anwendung von EU-Recht geltende Unabhängigkeitsanforderung auch für die Anwendung des nationalen Rechts. Theoretisch wird der Geltungsbereich der europäischen Anforderung auf jene Fälle beschränkt, wo EU-Recht anzuwenden ist. ${ }^{20}$ Praktisch impliziert es jedoch, dass die mitgliedstaatlichen Gerichte auch außerhalb der Anwendung von EU-Recht unabhängig sein sollen, da das EU-Recht und das nationale Recht von denselben Gerichten angewendet wird.

Ein weiteres Element des verfassungsrechtlichen Torsos Europas ist, dass die Mitgliedstaaten die vom Binnenmarktrecht gestatteten Ausnahmen nur dann nutzen können, wenn die durch öffentliches Interesse gerechtfertigte Handelsbeschränkung die europäischen Rechtsstaatlichkeitsanforderungen erfüllt. Die Beschränkung des Handels, auch wenn sie notwendig und verhältnismäßig (d.h. gerechtfertigt) ist, ist nicht akzeptabel, wenn sie das Rechtsstaatlichkeitsprinzip nicht beachtet. ${ }^{21}$ In Anbetracht dessen, dass der Geltungsbereich der vier Grundfreiheiten äußerst breit ist, ${ }^{22}$ bedeutet dies

20 EuGH, Associação Sindical (Fn. 19), Rn. 31-33. DEP.

21 EuGH, Urteil v. 18.6.1991, Rs. C-260/89, ECLI:EU:C:1991:254, Rn. 43 - ERT gegen

22 Mit der Ausnahme von Verkaufsmodalitäten, die nur dann verboten sind, wenn sie diskriminierend sind, siehe EuGH, Urteil v. 24.11.1993, Rs. C-267/91 und C-268/91, Slg. 1993, I-06097, ECLI:EU:C:1993:905, Rn. 16 - Keck und Mithouard, alle mitgliedstaatlichen Maßnahmen, die den grenzüberschreitenden Verkehr verhindern, gegen Binnenmarkrecht verstoßen, auch wenn sie nicht diskriminierend sind.Siehe z.B. EuGH, Urteil v. 4.6.2002, Rs. C-503/99, Slg. 2002, I-04809, ECLI:EU:C:2002:328 - Kommission gegen Belgien; EuGH, Urteil v. 23.5.2000, Rs. C-58/99, Slg. 2000, I-3811, ECLI:EU:C:2000:280 - Kommission gegen Italien; EuGH, Urteil v. 4.6.2002, Rs. C-367/98, Slg. 2002, I-4731, ECLI:EU: C:2002:326 - Kommission gegen Portugal; EuGH, Urteil v. 4.6.2002, Rs. C-483/99, Slg. 2002, I-4781, ECLI:EU:C:2002:327 - Kommission gegen Frankreich; EuGH, Urteil v. 2.6.2005, Rs. C-174/04, Slg. 2005, I-4933, ECLI:EU:C:2005:350 - Kommission gegen Italien; Catherine Barnard, Restricting Restrictions: Lessons for the EU from the US?, CLJ 68 (2009), 575-606 (575-576). 
praktisch, dass die europäischen Rechtsstaatlichkeitsanforderungen für alle mitgliedstaatlichen Maßnahmen gelten, welche die Wirtschaftstätigkeit beschränken. ${ }^{23}$

Darüber hinaus hat die Europäische Kommission gelegentlich ,juristische Kreativität“ gezeigt, um das Rechtsstaatsprinzip in den Mitgliedstaaten durchzusetzen. In diesen Fällen hat sich die Kommission auf die „unterstützenden Nebenwirkungen" von offenkundig nicht verbundenen Vorschriften berufen. ${ }^{24}$

Beispielsweise trat die Kommission im Fall Kommission gegen Ungarn ${ }^{25}$ gegen Ungarn auf, um die Unabhängigkeit der Justiz zu wahren. Obwohl diese Kreativität, nach dem Urteil des EuGH im Fall Portugiesische Richter, unnötig erscheint, derzeit war die Anwendbarkeit von Artikel 19 Abs. 1 AEUV zu diesem Zweck zweifelhaft. ${ }^{26}$ In diesem Fall senkte Ungarn das obligatorische Rentenalter für ungarische Richter erheblich und entfernte auf diesem Weg zahlreiche aktive Richter aus dem Amt. ${ }^{27}$ Die Kommission betrachtete diese Maßnahme unter dem Gesichtspunkt der Unabhängigkeit der Justiz als sehr bedenklich, weil die Massenentlassung von hochrangigen Richtern und die nachfolgende Massenrekrutierung von neuen Richtern der Regierung die Gelegenheit gab, den Personalbestand der Gerichte nachhaltig zu beeinflussen. Die Kommission stützte aber ihre Klage nicht auf die Unabhängigkeit der Justiz, sondern auf das Verbot der Altersdiskriminierung. Der Grund hierfür war, dass die Kommission Zweifel hatte, ob sie für die Wahrung der Unabhängigkeit der ungarischen Justiz zuständig war, deswegen stützte sie sich auf das Diskriminierungsverbot, für dessen Durchsetzung sie zuständig war. Obwohl die Kommission ihre Klage auf Diskriminierungsargumenten aufbaute, macht die Pressemitteilung über die Einleitung des Vertragsverletzungsverfahrens deutlich, dass es

23 Siehe z.B. EuGH, Urteil v. 11.6.2015, Rs. C-98/14, ECLI:EU:C:2015:386 - Berlington Hungary u.a.

24 Siehe Csongor István Nagy, Do European Union Member States Have to Respect Human Rights? The Application of the European Union's 'Federal Bill of Rights' to Member States, Ind. Int'l \& Comp. L. Rev. 27 (2017), 1-13 (9).

25 EuGH, Urteil v. 6.11.2012, Rs. C-286/12, ECLI:EU:C:2012:687 - Kommission gegen Ungarn.

26 Es ist anzumerken, dass der EuGH bereits in dieser Zeit unter Bezugnahme auf Artikel 2 auf die Unabhängigkeit der Justiz hätte zurückgreifen können. Siehe Gábor Halmai, The Early Retirement Age of the Hungarian Judges, in: Fernanda Nicola/Bill Davis (Hrsg.), EU Law Stories: Contextual and Critical Histories of European Jurisprudence, Cambridge: Cambridge University Press 2017, 471-488.

27 Unabhängigkeit von Zentralbank und Datenschutzbehörden, Maßnahmen im Justizwesen: Europäische Kommission leitet beschleunigte Vertragsverletzungsverfahren gegen Ungarn ein, Pressemitteilung der Europäischen Kommission (IP/12/24), (17.1.2012). 
bei dieser Rechtsstreitigkeit tatsächlich um die Unabhängigkeit der Justiz ging. ${ }^{28}$

Ein weiteres Beispiel für diesen Ansatz ist das Slowakische Sprachgesetz. ${ }^{29}$ Verschiedene Organisationen hatten auf negative menschenrechtliche Auswirkungen des Gesetzes hingewiesen. ${ }^{30}$ Die Kommission griff es aber an, weil es den freien Verkehr von Waren, Personen, Dienstleistungen und Kapital behinderte und daher gegen die Regeln des Binnenmarkts verstieß. Die Kommission stützte sich also auf die Spill-Over Effekte der Marktfreiheiten, um die Sprachenrechte der Minderheiten zu schützen. ${ }^{31}$

Der einzige Mechanismus des EU-Rechts, der eine echte diagonale Anwendung ermöglicht, ist Artikel $7 \mathrm{EUV}^{32} .{ }^{33}$ Hierbei handelt es sich aber um einen politischen Mechanismus, der kaum reguliert und deshalb rechtlich nutzlos ist. Obwohl Artikel 7 EUV den Rat ermächtigt, im Fall einer schwerwiegenden und anhaltenden Verletzung einzugreifen, kann ohne Einstimmigkeit keine bedeutende Sanktion auferlegt werden. ${ }^{34}$ Darüber hinaus, ermöglicht Artikel 7 EUV ausschließlich die Aussetzung einzelner Rechte des betroffenen Mitgliedstaates, sorgt aber nicht für die unmittelbare Anwendung des europäischen Rechtsstaatlichkeitsprinzips.

Obwohl Artikel 7 EUV oft als die „Atombombe“ des EU-Rechts bezeichnet wird, ist er in der Tat eher ein Sicherheitsventil, das aus drei Gründen lückenhaft ist. Erstens ist der Mechanismus, als Folge der Einstimmigkeitsanforderung, ${ }^{35}$ praktisch kaum anwendbar. ${ }^{36}$ Zweitens fehlt es ihm an wirk-

28 Pressemitteilung der Europäischen Kommission (IP/12/24), Unabhängigkeit von Zentralbank und Datenschutzbehörden, Maßnahmen im Justizwesen: Europäische Kommission leitet beschleunigte Vertragsverletzungsverfahren gegen Ungarn ein, 17.1.2012.

29 Gesetz über die Staatssprache der Slowakischen Republik (Gesetz Nr. 270/1995). Siehe Csongor István Nagy, Slowakisches Sprachengesetz gegen Binnenmarkt - oder wie der Provinzialismus den Handel schädigt, in: András Masát/Ellen Bos/Martina Eckardt/Georg Kastner/ David R. Wenger (Hrsg.), Der Donauraum in Europa, Baden-Baden: Nomos 2013, 402-412.

30 Siehe z.B. Europäische Kommission für Demokratie durch Recht (Venedig Kommission), Opinion on the Act on the State Language of the Slovak Republic, Opinion No. 555/ $2009(15 . / 16.10 .2010)$.

31 Siehe Opinion on the Implementing Principles to the Slovak State Language Law Prepared by the European Commission's Legal Service (2010), verfügbar unter <http:// www.hhrf.org $>$.

32 Siehe von Bogdandy/Ioannidis (Fn. 1), 291-294.

33 Abgesehen von einigen Ausnahmen. Siehe z. B. Artikel 157 TFEU.

34 Wojciech Sadurski, Adding Bite to a Bark: the Story of Article 7, E. U. Enlargement, and Jörg Haider, 16 Columbia Journal of European Law 16 (2010), 385-426 (388-389); Chronowski (Fn. 6), 16.

35 Sadurski (Fn. 34), 388-389.

36 Vgl. Leonard Besselink, The Bite, the Bark, and the Howl - Article 7 TEU and the Rule of Law Initiatives, in: Jakab/Kochenov (Fn. 1), 128-144 (134), “The main use of Article 7 was that actually using it is unthinkable." 
samen Rechtsfolgen, da er für die Opfer der konkreten Rechtsverletzungen keine Rechtsbehelfe bietet, sondern den Rat nur ermächtigt, eine Sanktion zu verhängen, ohne die Rechtsverletzung zu beheben. In der Tat kann die von dem Rat auferlegte Sanktion kontraproduktiv sein: während sie für die Opfer keinen Rechtsschutz ermöglicht, kann sie nationalen Ressentiments Vorschub leisten. Drittens ist Artikel 7 EUV ein genuin politischer Mechanismus, der einen sehr schwachen normativen Charakter aufweist. Ein solches Verfahren kann leicht zum Gegenstand von politisch motivierter Kritik oder gar nationalistischer Demagogie verkommen.

\section{Mehrebenen-Verfassungssysteme in rechtsvergleichen- der Perspektive}

Die vergleichende Föderalismusforschung bietet eine Reihe Einsichten, Lösungen und Techniken, um das Problem der diagonalen Anwendung des Rechtsstaatlichkeitsprinzips in den Griff zu bekommen und praktikable Lösungen zu finden. Das Spektrum der föderalen Lösungsansätze ist breit und reicht von unitarischen föderalen Grundrechtsschutzsystemen (z. B. Österreich, Belgien und Deutschland), die auf dem einheitlichen normativen Charakter der Grundrechte beruhen, bis zu Ländern mit einem ausgeprägten Pluralismus auch im Bereich der Grundrechte. Die verschiedenen föderalen Systeme bieten ein breites Spektrum an Lösungsmustern. In Kanada gilt die Charta der Rechte und Freiheiten gleichermaßen für die Bundesregierung wie für die Provinzen. ${ }^{37}$ In Australien enthält die Bundesverfassung keinen Grundrechtskatalog. Dazwischen stehen die Vereinigten Staaten, deren Verfassungsgeschichte die größte Ähnlichkeit zu derjenigen der europäischen Integration aufweist.

Der am stärksten vertikal ausgerichtete (und für die EU ungeeignete) Ansatz wird von unitarischen föderalen Grundrechtsschutzsystemen (z. B. Belgien, Deutschland und Österreich) geboten, die zwei wichtige Charakteristika vereinen. Einerseits beschränkt sich der föderale Grundrechtsschutz nicht auf Minimalstandards, sondern ist umfassend und erschöpfend geregelt. Obwohl die Gliedstaaten ihre eigenen Grundrechtsschutzsysteme haben können, die für die eigenen Angelegenheiten der Gliedstaaten gelten, zielt das Grundrechtsschutzsystem des Bundesstaates darauf ab, unabhängig und vollständig zu sein. Zweitens haben die vom Bundesrecht anerkannten Rechte einen einheitlichen Inhalt, der für örtliche Unterschiede keinen Raum lässt:

37 Kanadische Charta der Rechte und Freiheiten, Art. 32 Abs. (1). 
Die Gliedstaaten haben keinen Ermessensspielraum außerhalb der bundesgesetzlichen Vorgaben. ${ }^{38}$

Dieser einheitliche Ansatz könnte der Vielfalt in Europa keine Rechnung tragen. Erstens haben die Mitgliedstaaten zu den in Artikel 2 EUV genannten Grundwerten zwar zugestimmt, allerdings ist es offenkundig, dass sie diesen Teil ihrer Souveränität nicht aufgegeben und keine umfassende Befugnis im Menschenrechtsschutz auf die Union übertragen haben. Deswegen wären die Vereinheitlichung der Rechtsstaatlichkeitsanforderungen und deren unbeschränkte Anwendung auf die Mitgliedstaaten widersprüchlich. Zweitens sind die Wahrung der Verfassungsidentität der Mitgliedstaaten ${ }^{39}$ und die Subsidiarität ebenfalls Grundsätze des EU-Rechts. Zudem haben sie den gleichen Rang, wie andere europäische Kernwerte. Dieser Umstand beschränkt die Europäisierung. Artikel 4 Abs. 4 EUV bestimmt: „Die Union achtet die Gleichheit der Mitgliedstaaten vor den Verträgen und ihre jeweilige nationale Identität, die in ihren grundlegenden politischen und verfassungsmäßigen Strukturen einschließlich der regionalen und lokalen Selbstverwaltung zum Ausdruck kommt."

Im Gegensatz dazu, verwenden flexible föderale Grundrechtsschutzsysteme verschiedene Methoden, um die auf der Bundesebene anerkannten Rechte als „gemeinsame Werte“ zu schützen und gleichzeitig regionalen Identitäten Raum zu geben.

Die kanadische Charta der Rechte und Freiheiten findet sowohl horizontale als auch diagonale Anwendung und gilt gleichermaßen für die Bundesregierung wie für die Provinzen. ${ }^{40}$ Die Vereinheitlichung der kanadischen Grundrechte ist jedoch nicht so kraftvoll, wie es auf den ersten Blick erscheinen mag. Der Gedanke des Föderalismus beeinflusst die Rechtsprechung, ${ }^{41}$ und die Provinzen (sowie die Bundesregierung) haben das Recht, sich einseitig den Kernregeln der Charta zu entziehen. ${ }^{42}$

38 Siehe z. B. Kruzifix-Beschluss des deutschen Bundesverfassungsgerichts, 1 BvR 1087/91, BVerfGE 93, 1-37.

39 Siehe Monika Polzin, Verfassungsidentität. Ein normatives Konzept des Grundgesetzes?, Tübingen: Mohr Siebeck, 2018.

40 Kanadische Charta der Rechte und Freiheiten, Art. 32 Abs. (1).

41 James B. Kelly, Reconciling Rights and Federalism during Review of the Charter of Rights and Freedoms: The Supreme Court of Canada and the Centralization Thesis, 1982-1999, CJPS 34 (2001), 321-355; Jeremy A. Clarke, The Charter of Rights and a Margin of Appreciation for Federalism: Lessons from Europe. Vortrag bei der Canadian Political Science Association, York University, Toronto, Ontario, 1.6.2006 (Panel L-3), verfügbar unter <https:// www.cpsa-acsp.ca>.

42 Kanadische Charta der Rechte und Freiheiten, Art. 33. Siehe Ford v. Quebec (Attorney General), [1988] 2 S. C. R. 712, Rn. 33; Johanne Poirier, Legal Proceedings Available to Individuals before the Highest Courts: a Comparative Law Perspective (Canada), European Parliament Research Services, Brüssel, 2017, 37, verfügbar unter <http://www.europarl.europa.eu>. 
Artikel 33 der kanadischen Charta enthält die Derogationsklausel („disposition dérogatoire“, "notwithstanding clause“), der sowohl die Bundesregierung als auch die Provinzen berechtigt, die Anwendung der Kernregeln der Charta auszusetzen. Diese Vorschrift ermöglicht eine Derogation für fünf Jahre, sie kann jedoch so oft, wie die Bundesregierung oder die Provinz es wünscht, erneuert werden. Die Derogationsklausel betrifft den Kern der von der Charta festgelegten Grundrechte (Artikel 2 und Artikel 7-15). Artikel 2 der Charta listet die grundlegenden Freiheitsrechte, wie Gewissensfreiheit, Religionsfreiheit, Gedankenfreiheit, Meinungsfreiheit, Pressefreiheit, Versammlungsfreiheit und Vereinigungsfreiheit auf. Artikel 7-15 der Charta enthalten die Justizgrundrechte (Artikel 7-14) und die Gleichheitsrechte (Artikel 15). Die Rechte, die nicht Gegenstand einer Derogationserklärung sein können, sind die politischen Teilhaberechte, welche die Mitwirkung an der politischen Willensbildung und der demokratischen Regierung sicherstellen, die Mobilitätsrechte (die Freizügigkeit innerhalb Kanadas sowie das Recht, aus Kanada ein- und auszureisen und sich in einer beliebigen Provinz niederzulassen) und die Sprachenrechte, die sicherstellen, dass die Bürger mit der Bundesregierung und bestimmten Provinzen auf Englisch oder Französisch kommunizieren können.

Obwohl die Provinzen von der Derogationsklausel nur einige Male und nur vorübergehend Gebrauch gemacht haben (die Bundesregierung hat diese Möglichkeit noch nie genutzt), ${ }^{43}$ ist die darin festgelegte Derogationsbefugnis umfassend. Einerseits kann eine Provinz (und die Bundesregierung) die aufgrund der Derogationsklausel verkündete Aussetzung bestimmter Grundrechte so oft wie erwünscht erneuern (jedes Mal kann die Aussetzung nur für fünf Jahre verkündet werden). Zweitens erfordert die Derogationsklausel keine Spezifität: Während die Provinzen im Allgemeinen die Anwendung bestimmter Gesetze aussetzen, gab Quebec im Jahre 1982, unmittelbar nach der Verabschiedung der neuen Verfassung und der Charta, eine pauschale Erklärung ab, die alle québécois Gesetze von den entsprechenden Vorschriften der Charta entband. ${ }^{44}$ Der Oberste Gerichtshof von Kanada erklärte diese Pauschalderogation für verfassungsmäßig. ${ }^{45}$

Der Gedanke des Föderalismus wird in der Charta und der hierzu ergangenen Rechtsprechung an verschiedenen Stellen ersichtlich. Erstens enthält die Charta keine wirtschaftlichen oder sozialen Rechte, daher kann sie als ein Minimalstandard ${ }^{46}$ angesehen werden, der keinen umfassenden Menschen-

\footnotetext{
43 Poirier (Fn. 42), 37.

44 Act Respecting the Constitution Act, 1982.

45 Ford v. Quebec (Fn. 42).

46 Poirier (Fn. 42), 37.
} 
rechtsschutz vorsieht und der Gesetzgebung der Provinzen breiten Raum lässt. ${ }^{47}$ Zweitens sieht Artikel 27 vor, dass die Charta unter Berücksichtigung des multikulturellen Erbes der Kanadier ausgelegt werden muss. Schließlich hat die Rechtsprechung, obwohl das kanadische Verfassungsrecht keine spezifische Doktrin über die Beziehung zwischen der Charta und dem Föderalismus entwickelt hat, ${ }^{48}$ eine erhebliche Sensitivität hinsichtlich der Souveränität der Provinzen gezeigt. 49

Aus europäischer Sicht scheint der kanadische Föderalismus, sowohl bezüglich der Wahrung der regionalen Identitäten als auch hinsichtlich der Garantien der Effektivität, der europäischen Integration nicht angemessen zu sein. Die kanadische Föderalisierung der Grundrechte funktioniert aufgrund ihrer festen politischen Verankerung, die sich aus einem politischen Gesamtkonsens und der gesellschaftlichen Akzeptanz der Charta ergibt. ${ }^{50}$ Das in Artikel 33 verankerte politische Derogationsrecht wird nur punktuell und nie über einen langen Zeitraum ausgeübt. Es gibt aber nichts, was die Provinzen daran hindern würde, dieses Recht systematisch und dauerhaft in Anspruch zu nehmen.

Demgegenüber enthält das australische Verfassungsrecht keine explizite Lösung für das Verhältnis zwischen zentral- und gliedstaatlichem Grundrechtsschutz. Die Bundesverfassung Australiens enthält keinen Grundrechtskatalog und, von einigen Ausnahmen abgesehen, bestimmt sie weder horizontal noch diagonal anwendbare Grundrechte. Obwohl die Bundesverfassung einzelne Grundrechte normiert, von denen einige auch für die Bundesstaaten gelten, ${ }^{51}$ ergibt sich aus diesen vereinzelten Vorschriften kein umfassender Grundrechtsschutz. In der Praxis hat Australien politische (legislative) Antworten auf die möglichen Grundrechtskonflikte zwischen der zentral- und der gliedstaatlichen Ebene gegeben.

Zum Beispiel wurde die Kriminalisierung der homosexuellen Beziehungen im Gegensatz zu den Vereinigten Staaten ${ }^{52}$ und Europa ${ }^{53}$ nicht durch die Rechtsprechung, sondern durch ein Gesetz aufgehoben. Nachdem bei dem Menschenrechtsausschuss der Vereinten Nationen im Fall Toonen eine er-

47 Kanadische Charta der Rechte und Freiheiten, Art. 26.

48 Die kanadische Rechtswissenschaft ist von der Frage des Ermessensspielraums nicht völlig unberührt geblieben. Siehe Clarke (Fn. 41).

49 Kelly (Fn. 41).

50 Poirier (Fn. 42), 37.

51 Nicholas Aroney/James Stellios, Rights in the Australian Federation, in: Nagy (Fn. 3), 258-268.

52 Lawrence v. Texas, 539 U.S. 558 (2003).

53 EGMR, Dudgeon v. United Kingdom, Case No. 7525/76, Urteil v. 22.10.1981; EGMR, Norris v. Ireland, Case No. 10581/83, Urteil v. 26.10.1988); EGMR, Modinos v. Cyprus, Case No. 15070/89, Urteil v. 22.4.1993. 
folgreiche Beschwerde gegen die anti-homosexuellen Gesetze von Tasmanien eingelegt wurde, ${ }^{54}$ verabschiedete der Bundesgesetzgeber ein Gesetz, ${ }^{55}$ das die entsprechenden strafrechtlichen Vorschriften Tasmaniens entkräftete. ${ }^{56}$

Ein weiteres Beispiel ist das Euthanasiegesetz des Nordterritoriums von 1995, das die Euthanasie im Nordterritorium legalisierte. ${ }^{57}$ Nach zwei Jahren wurde das Gesetz des Nordterritoriums durch ein Bundesgesetz ${ }^{58}$ außer Kraft gesetzt. ${ }^{59}$

Trotz einer oberflächlichen Ähnlichkeit zum EU-Recht - in dem Sinne, dass es an einem umfassenden föderalen Grundrechtsschutz mangelt und politische Lösungen bevorzugt werden - zeigen sich bei genauerem Hinsehen bedeutende Unterschiede zwischen letzterem und dem australischen Verfassungsrecht. Erstens weisen die Bundesstaaten von Australien eine viel geringere kulturelle Unterschiedlichkeit auf als die Mitgliedstaaten der EU. In der Tat sind sie kulturell weitestgehend einheitlich. Z.B. ist das Privatrecht in Australien größtenteils einheitlich und allgemein anerkannt, da das Common Law Teil des Bundesrechts ist. Ein föderales Common Law besteht nicht einmal in den Vereinigten Staaten, ${ }^{60}$ wo die Common LawTradition nicht im Bundesrecht, sondern in den Privatrechtsordnungen der Bundesstaaten fortlebt, deshalb gibt es mehr als 50 Privatrechtssysteme. Zweitens hat die australische Bundesregierung viel weitergehende Regelungsbefugnisse als die EU, die politische Lösungen ermöglichen. Gemäß Artikel 122 der australischen Bundesverfassung sind ferner die Territorien (aber nicht die Bundesstaaten) dem australischen Parlament und der australischen Regierung unterstellt.

Das derzeitige System der EU entspricht am stärksten dem US-amerikanischen Verfassungsrecht der ersten anderthalb Jahrhunderte: Obwohl heute, aufgrund der Inkorporationstheorie, die Mehrheit der für die Bundesregierung geltenden Grundrechte (aber nicht alle!) auch für die Bundesstaaten gelten, ${ }^{61}$ herrschte in den ersten anderthalb Jahrhunderten der Geschichte der Vereinigten Staaten die Meinung, dass - von den wenigen in der Verfassung

54 Toonen v. Australia, Communication No. 488/1992, U.N. Doc CCPR/C/50/D/488/ 1992 (1994).

55 Human Rights (Sexual Conduct) Act 1994 (Cth).

56 Siehe Katharine Gelber, Treaties and Intergovernmental Relations in Australia: Political Implications of the Toonen Case, AJPIL 45 (1999), 330-345.

57 Rights of the Terminally Ill Act 1995.

58 Euthanasia Laws Act 1997 (Cth).

59 Das Gesetz wiederrief die Befugnis des Nordterritoriums über die Frage. Section 50A of the Northern Territory (Self-Government) Act 1978.

60 Erie Railroad Co. v. Tompkins, 304 U.S. 64 (1938).

61 Jacqueline R. Kanovitz, Constitutional Law, 12. Aufl., Burlington: Elsevier Science 2010, 23. 
ausdrücklich genannten Rechten abgesehen ${ }^{62}$ - der in den ersten zehn Zusatzartikeln untergebrachte umfassende Grundrechtskatalog (die Bill of Rights) gegenüber den Bundesstaaten keine Anwendung findet. Die verfassungsrechtliche Erfahrung, die zur Umwälzung dieses Systems führte, war die Erkenntnis, dass das föderale System ohne die Wahrung der gemeinsamen Grundwerte nicht überlebensfähig ist.

Ursprünglich enthielt die amerikanische Verfassung keinen Grundrechtskatalog: Bei der verfassunggebenden Versammlung wurde ein einschlägiger Vorschlag abgelehnt. Bei den Ratifizierungsverfahren in den Bundesstaaten ergab sich jedoch Widerstand gegen die Bundesverfassung. Das Argument war, dass die Bundesstaaten der Bundesregierung keine Macht ohne Beschränkung durch Grundrechte übertragen könnten. Die ersten zehn Zusatzartikel wurden verabschiedet, um die Ratifizierung der Verfassung sicherzustellen. ${ }^{63}$ Dieser historische Hintergrund macht das ursprüngliche Ziel der Bill of Rights deutlich. Die Anwendung der Bill of Rights auf die Gliedstaaten wurde zunächst überhaupt nicht in Betracht gezogen. Der Oberste Gerichtshof machte das sehr deutlich im Fall Barron gegen Baltimore, ${ }^{64}$ als er feststellte, dass der Grundrechtskatalog der Bundesverfassung ausschließlich auf die Bundesregierung Anwendung findet, während umgekehrt die Grundrechtskataloge der einzelstaatlichen Verfassungen ausschließlich auf die betreffenden Bundesstaaten anwendbar sind, und keine diagonale Anwendung finden.

In dieser Hinsicht stellte der nach dem Bürgerkrieg verabschiedete Zusatzartikel XIV einen Wendepunkt dar, jedoch sollte es auch danach noch Jahrzehnte dauern, bis die Rechtsprechung diesen Paradigmenwechsel widerspiegelte. Anderthalb Jahrhunderte waren die Bundesstaaten ausschließlich den Grundrechten der eigenen Verfassungen unterworfen. ${ }^{65}$

Um die Abschaffung der Sklaverei als Ergebnis des Bürgerkriegs zu sichern, erklärte der Zusatzartikel XIV die Anwendbarkeit einiger (doch bei weitem nicht aller) Bundesgrundrechte auf die Bundesstaaten. Er bezweckte aber nicht die Schaffung eines umfassenden Grundrechtssystems auf Bundesebene.

62 Siehe Artikel I Absatz 10 der US-Bundesverfassung: „Kein Einzelstaat darf [...] ein Ausnahmegesetz, das eine Verurteilung ohne Gerichtsverfahren zum Inhalt hat, oder ein Strafgesetz mit rückwirkender Kraft oder ein Gesetz, das Vertragsverpflichtungen beeinträchtigt, verabschieden oder einen Adelstitel verleihen." Christian Lammert/Markus B. Siewert/Boris Vormann (Hrsg.), Handbuch Politik USA, 1. Aufl., Berlin/Heidelberg: Springer 2016, 683-708.

63 Siehe Kenneth $R$. Stevens, Perspectives on Comparative Federalism: The American Experience in the Pre-Incorporation Era, in: Nagy (Fn. 3), 120-128 (122).

6432 U.S. (7 Pet.) 243, 247-248 (1833).

65 Barron v. Baltimore, 32 U.S. (7 Pet.) 243 (1833). 
„Keiner der Einzelstaaten darf Gesetze erlassen oder durchführen, die die Vorrechte oder Freiheiten von Bürgern der Vereinigten Staaten beschränken, und kein Staat darf irgendjemandem ohne ordentliches Gerichtsverfahren nach Recht und Gesetz Leben, Freiheit oder Eigentum nehmen oder irgendjemandem innerhalb seines Hoheitsbereiches den gleichen Schutz durch das Gesetz versagen. “66

Die Doktrin der parallelen Grundrechtsschutzsysteme schlug so tiefe Wurzeln, dass die Rechtsprechung ein halbes Jahrhundert lang ablehnte, den anderen föderalen Grundrechten gegen die Staaten Geltung zu verschaffen. 1876, im Fall United States gegen Cruikshank, entschied der Oberste Gerichtshof, dass das im ersten Zusatzartikel verankerte Versammlungsrecht die Befugnisse der Regierungen der Bundesstaaten in Bezug auf ihre eigenen Bürger nicht beschränkt, beziehungsweise, dass der erste Zusatzartikel ausschließlich für die Bundesregierung gilt. 67

1897 entschied der Oberste Gerichtshof im Fall Chicago, Burlington $\mathcal{E}$ Quincy Railroad Co. gegen Chicago, dass die Anforderung einer fairen Entschädigung im Fall von Enteignung aufgrund der Due Process-Klausel auch für die Bundesstaaten gilt. ${ }^{68}$ Rückblickend kann dieses Urteil als das erste angesehen werden, in dem der Oberste Gerichtshof ein Bundesgrundrecht gegen die Bundesstaaten für anwendbar erklärt hat. Da Zusatzartikel XIV den Schutz des Eigentums hinsichtlich des Schutzbereiches der Due ProcessKlausel ausdrücklich erwähnt („,kein Staat darf irgendjemandem ohne ordentliches Gerichtsverfahren nach Recht und Gesetz [...] Eigentum nehmen“), war jedoch noch ungewiss, ob die Anwendung des föderalen Eigentumsschutzes gegen die Bundesstaaten unmittelbar aus dem vierzehnten Zusatzartikel folgte oder die Bestimmung als Einfallstor der in den ersten zehn Zusatzartikeln verankerten Bill of Rights diente.

Der eigentliche Durchbruch folgte 1925 mit dem Fall Gitlow gegen New York, ${ }^{69}$ in dem der Oberste Gerichtshof die Anwendbarkeit der Bundesgrundrechte gegenüber den Einzelstaaten explizit bejahte (in diesem Fall unter ausdrücklicher Bezugnahme auf den Ersten Zusatzartikel). ${ }^{70}$ Es folgten zahlreiche Fälle, in denen die Anwendung der Bundesgrundrechte auf die Bundesstaaten ausgeweitet wurde. Der Oberste Gerichtshof inkorporierte

66 Lammert/Siewert/Vormann (Fn. 62), 683-708.

67 United States v. Cruikshank, 92 U. S. 542, 552 (1876).

68 Chicago, B. E Q. R. Co. v. Chicago, 166 U.S. 226 (1897).

69 Gitlow v. New York, 268 U.S. 652 (1925).

$70 \mathrm{Vgl}$. Stanley Morrisona, Does the Fourteenth Amendment Incorporate the Bill of Rights?, Stanford L. Rev. 2 (1949), 140-173 (152), "The assertion of th[e] [substantive due process] doctrine, incidentally, gave to the Fourteenth Amendment an importance vastly greater than it was supposed to have in 1868. But the development of substantive due process is a story far removed from the question of incorporation of the Bill of Rights." 
die Bundesgrundrechte Schritt für Schritt in das Recht der Bundesstaaten und formulierte ein auf dem Konzept der Kernstandards beruhendes Trennungsprinzip. Im Fall Snyder gegen Massachusetts ${ }^{71}$ legte der Oberste Gerichtshof fest, dass „es den Staaten freisteht, das Verfahren seiner Gerichte nach seiner eigenen Auffassung von Politik und Gerechtigkeit zu regeln, es sei denn, das verstößt gegen einen Grundsatz der Gerechtigkeit, der so in den Traditionen und im Gewissen unseres Volkes verwurzelt ist, dass er als grundlegend eingestuft wird“. Im Fall Palko gegen Connecticut sprach er dasselbe Prinzip aus und entschied, dass für die Bundesstaaten nur jene Bundesgrundrechte gelten, die „das Wesentliche eines Systems geordneter Freiheit" bedeuten. ${ }^{72}$ Obwohl der Gerichtshof nun die Mehrheit (jedoch nicht alle!) der in den ersten zehn Zusatzartikeln normierten Rechte gegenüber den Bundesstaaten für anwendbar erklärte, zeigt die Epoche nach dem Bürgerkrieg, wie das Gericht durch die selektive Inkorporation der Bundesgrundrechte versuchte, ${ }^{73}$ die diagonal anwendbaren Rechte von den ausschließlich für die Bundesregierung geltenden Rechten zu trennen.

Es ist keine Übertreibung zu sagen, dass der Zusatzartikel XIV sehr erfolgreich als Grundlage eines umfassenden föderalen Grundrechtsschutzes diente und damit die Verbindung zwischen dem Zentralstaat und den Bürgern stärkte. Es ist bemerkenswert, dass es nicht die Politik und pragmatische politische Lösungen, sondern die Gerichte waren, die dieses Defizit der Rechtsstaatlichkeit beseitigten. Obwohl der vierzehnte Zusatzartikel sowohl einen judikativen als auch einen gerichtlich kontrollierten politischen Mechanismus enthält, spielten die Gerichte in diesem Prozess die zentrale Rolle. Der erste Abschnitt des vierzehnten Zusatzartikels listet einige ausgewählte und gerichtlich durchsetzbare Rechte auf, die für diagonal anwendbar erklärt werden, während Abschnitt 5 den Kongress ermächtigt „die Bestimmungen dieses Zusatzartikels durch entsprechende Gesetze zur Geltung zu bringen“. Beide Abschnitte unterliegen gerichtlicher Kontrolle. Abschnitt 1 kommt in Individualrechtsstreitigkeiten zur Anwendung, während die Ausübung der von Abschnitt 5 übertragenen Gesetzgebungsbefugnis vom Obersten Gerichtshof kontrolliert wird: Der Kongress darf keine neuen Rechte einführen und den Inhalt der aufgelisteten Rechte nicht erweitern. Seine Befugnis beschränkt sich auf die Durchsetzung dieser Rechte, und das Erfordernis einer „angemessenen Gesetzgebung “ impliziert, dass das verabschiedete Bundesgesetz verhältnismäßig sein und einen Rechtsschutzzweck verfolgen muss. ${ }^{74}$

71 Snyderv. Massachusetts, 291 U. S. 97, 105 (1934).

72 Palko v. Connecticut, 302 U.S. 319, 325 (1937).

73 Otis H. Stephens Jr./John M. Scheb II, American Constitutional Law. Volume II: Civil Rights and Liberties, Andover (Hampshire): Cengage Learning 2007, 23-24.

74 City of Boerne v. Flores, 521 U.S. 507, 519 (1997). 
Es gibt wichtige Ähnlichkeiten zwischen den ersten eineinhalb Jahrhunderten des Bundessystems der Vereinigten Staaten und dem EU-Recht.

In beiden Systemen war der Grundrechtskatalog (die Bill of Rights in den ersten zehn Zusatzartikeln der amerikanischen Verfassung und die Charta der Grundrechte der Europäischen Union) Ausdruck derselben Überlegung (dass ohne Beschränkung durch Grundrechte keine öffentliche Macht existieren darf) und wurde ursprünglich geschaffen, um die zentrale Regierung in Schranken zu weisen. In keiner der Verfassungen gab es das Bestreben, ein föderales Grundrechtsschutzsystem für die Gliedstaaten zu konzipieren. In beiden Fällen konzentrierten sich die Überlegungen auf die Schaffung einer funktionsfähigen und rechtsstaatlichen Exekutive auf der (quasi-)föderalen Ebene.

In den Vereinigten Staaten wurde das Einfügen einer Bill of Rights in die Bundesverfassung bei der Verfassungskonvention abgelehnt, aber bei der Ratifizierung der Bundesverfassung mehrten sich die kritischen Stimmen. Der Einwand war, dass ohne Beschränkung durch Grundrechte keine Macht existieren darf. Deshalb wurden die ersten zehn Zusatzartikel zur Bundesverfassung angenommen: Es wurde zunehmend klarer, dass die Ratifizierung der Bundesverfassung ohne die Annahme einer solchen Bill of Rights scheitern könnte. ${ }^{75}$

Die EU-Charta ist durch einen ähnlichen Prozess gegangen. Der Vorgänger der Charta waren die allgemeinen Rechtsgrundsätze des Gemeinschaftsrechts zum Schutze der Grundrechte: Wo Macht übertragen wird, muss auch deren Beschränkung durch die Grundrechte vorgeschrieben werden, da diese zwei Aspekte untrennbar miteinander verbunden sind. Für den EuGH war diese Verbindung so grundlegend, dass er sie in Gestalt der allgemeinen Rechtsgrundsätze in die Gemeinschaftsrechtsordnung integrierte. Es ging dabei jedoch keineswegs um eine Grundrechtskontrolle über die Mitgliedstaaten. ${ }^{76}$ Die vom Gericht entwickelten Grundrechtsanforderungen fanden später ihren Weg in die Charta, die ebenfalls nicht als allgemeines Grundrechtsschutzregime, sondern als ein Kontrollmechanismus der EU-Exekutive vorgesehen war. ${ }^{77}$ Diese Auffassung prägt den Geltungsbereich der Charta. Die Anwendung der Charta auf die Mitgliedstaaten bei der Durchsetzung des EU-Rechts trägt der besonderen institutionellen Verfasstheit der EU Rechnung. Da die EU keine regionalen Verwaltungsorgane hat, spielen die Mitgliedstaaten eine tragende Rolle bei der Durchsetzung des EU-Rechts.

75 Siehe Stevens (Fn. 63), 122.

76 Siehe Eeckhout (Fn. 15), 958-969.

77 Siehe Fontanelli (Fn. 16). 
Wenn sie als Beauftragte der EU auftreten, sind ihre Handlungen der EU zuzurechnen.

Die Verfassungsgeschichte der Vereinigten Staaten zeigt, dass die Anwendung der föderalen Grundrechte auf die Bundesstaaten vom Gedanken bestimmt wurde, dass deren Verletzung einen „Scheidungsgrund“ darlegen kann. Der amerikanische Bürgerkrieg beweist, dass es bestimmte Grundwerte gibt, die in der gesamten Union respektiert werden müssen, anders kann die Union nicht aufrechterhalten werden. Diese Erkenntnis führte zur Verabschiedung des vierzehnten Zusatzartikels, welcher die Anwendbarkeit einiger föderaler Grundrechte auf die Bundesstaaten vorsah. Interessanterweise war die Idee, dass die föderalen und die bundesstaatlichen Grundrechtsschutzregime parallel zueinander sind, so tief im amerikanischen Verfassungsdenken verankert, dass die amerikanischen Gerichte die Anwendung der föderalen Grundrechte auf die Bundesstaaten ein halbes Jahrhundert lang ablehnten. ${ }^{78}$

\section{Die Lehre von der Diagonalität}

Im Folgenden werden die ontologischen, dogmatischen und institutionellen Aspekte der hier vorgeschlagenen Lehre von der Diagonalität vorgestellt.

\section{Die ontologische Ebene: Warum ist die Diagonalitätslehre erforderlich?}

Die Ontologie der horizontalen Anwendung des Rechtsstaatlichkeitsprinzips ist klar: In einer demokratischen Gesellschaft kann keine öffentliche Gewalt ohne konstitutionelle Beschränkungen existieren. Die Wurzeln dieses Gedankens gehen auf das Zeitalter der Aufklärung zurück. ${ }^{79}$ Die Philosophie der Aufklärung ersetzte das von Gott gegebene Recht durch das Naturrecht und formulierte die Notwendigkeit konstitutioneller Beschränkungen des Gebrauchs öffentlicher Macht. Nach der Gesellschaftsvertragstheorie übertrugen die Menschen einen Teil ihrer Rechte auf den Staat (oder hätten sie die übertragen, wenn sie einen Gesellschaftsvertrag abgeschlossen hätten), ${ }^{80}$ be-

78 United States v. Cruikshank, 92 U. S. 542, 552 (1876).

79 Siehe z.B.St. Thomas Aquinas, Summa Theologiae 1-2, q. 96, a. 5 (Antwort auf den zweiten Einwand).

80 Jean-Jacques Rousseau, Le contrat social ou Principes du droit politique, Amsterdam: Chez Marc-Michel Rey 1762. 
wahrten aber ihre unveräußerlichen Rechte. ${ }^{81}$ Danach sind die Staatsgewalt und die Menschenrechte (die demokratische Kontrolle) von Natur aus miteinander verbunden.

Dies gibt jedoch keine Antwort auf die Frage, warum die föderalen Rechtsstaatlichkeitsanforderungen diagonal anwendbar sein sollten. Da die EU auf die Mitgliedstaaten keine souveräne Gewalt überträgt, kann sie sie beim Gebrauch ihrer öffentlichen Gewalt auch nicht beschränken. Ganz im Gegenteil, es sind die Mitgliedstaaten, die dafür sorgen müssen, dass die EU die auf sie übertragenen Befugnisse im Einklang mit den Rechtsstaatlichkeitsanforderungen ausübt. Die Geschichte der durch Vereinigung zustande gekommenen Bundesstaaten veranschaulicht dies. ${ }^{82}$

Die diagonale Anwendung des europäischen Rechtsstaatlichkeitsprinzips beruht sowohl auf theoretischen, als auch auf praktischen Gründen.

Einerseits ist das Rechtsstaatlichkeitsprinzip Teil der europäischen Identität. Das bedeutet, dass die Mitgliedstaaten die Einhaltung der gemeinsamen Regeln, einschließlich der gemeinsamen Werte der EU gewährleisten sollen. Es sind mit anderen Worten die Grundwerte, die die EU zusammenhalten, daher stellt der Verstoß gegen diese einen "Scheidungsgrund“ dar.83 Der Wortlaut von Artikel 2 AEUV macht dies deutlich, indem er diese Werte als das Fundament der Union bezeichnet. Diese Formulierung impliziert, dass bei ihrer Nichtbeachtung die gesamte Struktur zusammenbricht. Der Präambel der Charta nach gehören „die unverletzlichen und unveräußerlichen Rechte des Menschen sowie Freiheit, Demokratie, Gleichheit und Rechtsstaatlichkeit“ zu „dem kulturellen, religiösen und humanistischen Erbe Europas“. Im Lichte dieser Überlegungen, können die inneren Angelegenheiten eines Mitgliedstaates nur dann zu einer gemeinsamen Sache erklärt werden und kann die EU eine eigene Zuständigkeit nur insoweit für sich in Anspruch nehmen, als die identitätsbildenden Grundwerte der EU gewahrt werden müssen. Der eigentliche Grund, weshalb die Diagonalität eine der Kernfragen der europäischen Integration geworden ist, ist nicht das Fehlen einer europäischen Befugnis, die europäischen Grundwerte in den Mitgliedstaaten durchzusetzen, sondern die Tatsache, dass die Gemeinschaft diese Werte für grund-

\footnotetext{
81 Siehe z. B. John Locke, Two Treatises of Government I, Para. 63, London: Thomas Tegg 1823.

82 Bezüglich Australien, siehe Nicholas Aroney, The Constitution of a Federal Commonwealth: The Making and Meaning of the Australian Constitution, Cambridge: Cambridge University Press 2009, Kapitel 5, bezüglich der Vereinigten Staaten, siehe Stevens (Fn. 63), 122.

$83 \mathrm{Vgl}$. Closa/Kochenov/Weiler (Fn. 9), 5 ("The normative argument on the universal effects of the individual Member States' departures from the values of the Union on whole of the EU starts with the all-affected principle, related to the deep inter-penetration and the mutual interdependency between the Member States of the Union at the current stage of European integration.”).
} 
legend erklärt hat und zu einem Trennungsgrund im Falle der Nichtbeachtung durch einen Mitgliedstaat.

Im Hinblick auf die vorstehenden Erwägungen ist die diagonale Anwendung des europäischen Rechtsstaatlichkeitsprinzips nur insoweit gerechtfertigt, als es für die Wahrung der gemeinsamen europäischen Identität erforderlich ist. Diese Einsicht muss den Umfang der diagonalen Anwendung prägen. Es gibt keinen Grund, dem europäischen Rechtsstaatlichkeitsprinzip in diagonaler Richtung einen ebenso einheitlichen und erschöpfenden Inhalt zu geben wie in horizontaler Richtung. Die diagonale Anwendung hat einen anderen Zweck: Die Schaffung eines „Werteminimums“, das die zentrifugalen Kräfte im Zaum halten kann.

Andererseits sind die Kontrolle und Durchsetzung der europäischen Grundwerte nicht nur aufgrund theoretischer, sondern auch aufgrund praktischer Erwägungen angezeigt. Denn wenn die Mitgliedstaaten die aus den Grundwerten folgenden Anforderungen nicht erfüllen, kann dies die Funktionsunfähigkeit der gesamten EU in Mitleidenschaft ziehen. So enthält das EU-Recht verschiedene Kooperationsmechanismen, die auf dem gegenseitigen Vertrauen zwischen den Mitgliedstaaten beruhen (z. B. justizielle Zusammenarbeit in Zivil- und Strafsachen). Diese würden zusammenbrechen, wenn das gegenseitige Vertrauen in der EU nicht bewahrt werden könnte.

Die gegenseitige Anerkennung der Urteile, die Rechtshilfe und die Auslieferung im europäischen Justizraum sind nur dann funktionsfähige Mechanismen, wenn die Kernwerte in der ganzen Union beachtet werden. Die Anerkennung der Urteile kann nur sichergestellt werden, wenn die mitgliedstaatlichen Gerichte einander ohne Vorbehalt vertrauen. Man kann von den mitgliedstaatlichen Behörden nicht erwarten, Auslieferungsanträge routinemäßig stattzugeben, wenn sie - auch nur geringe - Zweifel haben, ob das Recht des Angeklagten auf ein faires Verfahren respektiert werden wird. ${ }^{84}$

Wie der EuGH im Fall Portugiesischer Richter betont hat:

„30 Nach Art. 2 EUV gründet sich die Union auf Werte wie die Rechtsstaatlichkeit, die allen Mitgliedstaaten in einer Gesellschaft, die sich u. a. durch Gerechtigkeit auszeichnet, gemeinsam sind. Das gegenseitige Vertrauen zwischen den Mitgliedstaaten und insbesondere zwischen deren Gerichten beruht auf der Prämisse, dass die Mitgliedstaaten eine Reihe gemeinsamer Werte teilen, auf die sich, wie es in Art. 2 EUV heißt, die Union gründet.“

Darüber hinaus beruht das Justizsystem der EU auf der Unabhängigkeit und Verlässlichkeit der mitgliedstaatlichen Gerichte und deren Bereitschaft,

84 EuGH, Urteil v. 25.7.2018, Rs. C-216/18 PPU, ECLI:EU:C:2018:586 - Minister for Justice and Equality (Mängel des Justizsystems) gegen LM. 
im Rahmen des Vorabentscheidungsverfahrens dem EuGH Auslegungsfragen vorzulegen. ${ }^{85}$

Schließlich hat die EU keine regionalen oder örtlichen Organe und muss sich auf die Hilfe der mitgliedstaatlichen Behörden verlassen. Die dezentrale Durchsetzung des EU-Wettbewerbsrechts ist ein gutes Beispiel. Nachdem sie erkannt hat, dass es ihr an den institutionellen Kapazitäten mangelt, EUWettbewerbsrecht in der ganzen Union durchzusetzen, hat die Kommission ein System dezentraler Durchsetzung entwickelt, um die mitgliedstaatlichen Wettbewerbsbehörden (und deren Durchsetzungskapazitäten) einzubeziehen. ${ }^{86}$ Dieses dezentrale System würde die Durchsetzung von EU-Wettbewerbsrecht indes nicht stärken, sondern schwächen, wenn die mitgliedstaatlichen Behörden unter politischem Einfluss stünden und die mitgliedstaatlichen Gerichte nicht dafür sorgen würden, dass den Unternehmen ein wirksamer Rechtschutz zur Verfügung steht. ${ }^{87}$

Die Ontologie der diagonalen Anwendung prägt die Funktionsweise der Diagonalitätslehre. Wenn das europäische Rechtsstaatlichkeitsprinzip auf die EU und auf die in deren Namen handelnden Mitgliedstaaten angewendet wird, gibt es keinen Grund, die europäischen Anforderungen nicht in vollem Umfang durchzusetzen. Wenn aber diese Anforderungen diagonal durchgesetzt werden, muss berücksichtigt werden, dass diese Richtung der Anwendung einen anderen $Z$ weck hat: Es muss sichergestellt werden, dass die Identität der Union und das gegenseitige Vertrauen zwischen den Mitgliedstaaten bewahrt werden. ${ }^{88}$ Mit anderen Worten: Während die horizontale Anwendung die uneingeschränkte Verwirklichung der Grundwerte bezweckt, begnügt sich die diagonale Anwendung mit dem gerade noch erträglichen (Mindest-) Niveau ihrer Umsetzung.

Dieselbe Schlussfolgerung ergibt sich, wenn die Frage von der Seite der Zuständigkeitsübertragung angegangen wird. Einerseits wäre es widersprüchlich, die europäischen Rechtsstaatlichkeitsanforderungen auf die Mitglied-

85 Sacha Prechal, National Courts in EU Judicial Structures, YBEL 25 (2006), 429-450.

$86 \mathrm{VO} / 1 / 2003 / \mathrm{EG}$ v. 16.12.2002 zur Durchführung der in den Artikeln 81 und 82 des Vertrags niedergelegten Wettbewerbsregeln. ABl. 2003 L 1, 1-25.

87 Siehe z. B. den ungarischen Wassermelone-Fall. Csongor István Nagy, Competition Law in Hungary, Zuidpoolsinge: Kluwer 2016, 35-37. Diese Überlegungen haben in der Verabschiedung der ECN+ Richtlinie eine Rolle gespielt. Siehe RL 2019/1/EU v. 11.12.2018 zur Stärkung der Wettbewerbsbehörden der Mitgliedstaaten im Hinblick auf eine wirksamere Durchsetzung der Wettbewerbsvorschriften und zur Gewährleistung des reibungslosen Funktionierens des Binnenmarkts. ABl. 2019 L 11, 3-33.

88 Vgl. Schlussanträge des Generalanwalts Maduro in EuGH, Urteil v. 31.1.2008, Rs. C-380/ 05, ECLI:EU:C:2007:505, Rn. 20 - Centro Europa, "Die Grundrechte müssen auf nationaler Ebene zwar nicht in genau demselben Maß geschützt sein wie auf der Ebene der Europäischen Union, doch muss ein gewisses Maß an Gleichwertigkeit bestehen, damit sichergestellt ist, dass das Recht der Union wirksam in der nationalen Rechtsordnung angewandt werden kann.” 
staaten in vollem Umfang, d. h. auch im Hinblick auf ihre eigenen Angelegenheiten, anzuwenden, weil sie diesen Teil ihrer Souveränität nie völlig übertragen und sich nie einem umfassenden europäischen Grundrechtsschutz unterworfen haben. Andererseits ist es offenkundig, dass die Mitgliedstaaten tatsächlich Versprechen hinsichtlich der Rechtsstaatlichkeit abgegeben haben (siehe Artikel 2 AEUV), ${ }^{89}$ deshalb ist es auch unstrittig, dass das europäische Rechtsstaatlichkeitsprinzip in den Mitgliedstaaten, zumindest in einem beschränkten Umfang, durchzusetzen ist.

Diese Erwägungen können bei der Durchsetzung des europäischen Rechtsstaatlichkeitsprinzips auf zweifache Weise berücksichtigt werden. Erstens verlangt die Ontologie der Diagonalität nicht, dass jede europäische Rechtsstaatlichkeitsanforderung auch auf die Mitgliedstaaten Anwendung findet. Ähnlich wie die Doktrin der selektiven Inkorporation, die in den Vereinigten Staaten zur Rechtfertigung der diagonalen Anwendung der Bill of Rights herangezogen wird, verlangen die EU-Grundwerte nicht die vollständige Inkorporation der europäischen Rechtsstaatlichkeitsanforderungen in das Recht der Mitgliedstaaten, sondern nur die diagonale, d.h. abgeschwächte Durchsetzung der föderalen Grundwerte. Wenn danach bestimmte Anforderungen der Rechtsstaatlichkeit auf einen Mitgliedstaat diagonale Anwendung finden, müssen die Mitgliedstaaten einen Ermessensspielraum (marge d'appreciation, margin of appreciation) haben. Die strukturellen Grundsätze des EU-Rechts erfordern, dass der Schutz der Grundrechte in den Mitgliedstaaten wirksam aber zugleich verhältnismäßig sein soll, d.h. er muss die nationalen Verfassungsidentitäten berücksichtigen. ${ }^{90}$ Als Beispiel: Für die irische Verfassungsidentität spielen die katholischen Traditionen eine zentrale Rolle; ${ }^{91}$ obwohl die gleichgeschlechtlichen Ehen in zahlreichen Mitgliedstaaten gültig sind, sind sie in vielen anderen Staaten (z. B. Polen, ${ }^{92}$ Slowakei, ${ }^{93}$ Ungarn ${ }^{94}$ ) nicht verfassungsgemäß; es gibt in den früheren sozia-

89 Siehe von Bogdandy/Ioannidis (Fn. 1), 287-291.

90 Siehe Bernhard Schima, EU Fundamental Rights and Member State Action after Lisbon: Putting the ECJ's Case Law in its Context, 38 Fordham Int'l L. J. 38 (2015), 1097-1133, (11131114).

91 Gary Jeffrey Jacobsohn, Constitutional Identity, Cambridge MA: Harvard University Press 2010, 188-190, 259-261, 350-351.

92 Artikel 18 der Verfassung der Republik Polen (2.4.1997), verfügbar unter <http:// www.sejm.gov.pl>.

93 Constitutional Act No. 161/2014 of the Collection of Laws, Art. 1, Abs. 1 v. 4.6.2014. Siehe Marián Sekerák, Same-Sex Marriages (or Civil Unions/Registered Partnerships) in Slovak Constitutional Law: Challenges and Possibilities, Utrecht Law Review 13 (2017), 34-59 (35).

94 Artikel L(1) des ungarischen Grundgesetzes (25.4.2011) (“Ungarn schützt die Institution der Ehe als eine aufgrund einer freiwilligen Entscheidung zwischen Mann und Frau zustande gekommene Lebensgemeinschaft sowie die Familie als Grundlage des Fortbestands der Nation."). 
listischen Mitgliedstaaten eine besondere Sensibilität gegenüber dem roten Stern, ${ }^{95}$ die in den westlichen Mitgliedstaaten viel geringer ist. Natürlich darf der Kern des Grundrechtsschutzes keine Frage örtlicher Gegebenheiten sein und der Kern dieser Rechte darf mit Verweis auf die konstitutionellen Identitäten nicht verletzt werden.

Die Charta enthält Rechte, besonders die im Titel IV aufgeführten wirtschaftlichen und sozialen Rechte, ${ }^{96}$ deren uneingeschränkte Anwendung, auf die Mitgliedstaaten im Bereich ihrer eigenen Angelegenheiten im Hinblick auf die europäische Vielfalt nicht zwingend sind und deren Inkorporation deshalb nicht gerechtfertigt ist. Andererseits können die europäischen Anforderungen an die Rechtsstaatlichkeit und die mitgliedstaatlichen Verfassungsidentitäten in bestimmten Fällen auch weitgehend übereinstimmen. Die Behandlung gleichgeschlechtlicher Ehen in der Rechtsprechung des Europäischen Gerichtshofs für Menschenrechte ist hierfür ein Beispiel. Im Fall Obergefell gegen Hodges hat der Oberste Gerichtshof der USA festgestellt, dass alle Staaten die gleichgeschlechtlichen Ehen anerkennen müssen. ${ }^{97}$ Der Europäische Gerichtshof für Menschenrechte vertritt in diesem Punkt eine differenziertere Auffassung. Er hat festgestellt, dass der Status der gleichgeschlechtlichen Paare anerkannt werden muss, aber die Staaten müssen diese Beziehungen nicht Ehe nennen. Mit diesem Kompromiss hat der Europäische Gerichtshof für Menschenrechte für einen der Ehe mehr oder weniger ähnlichen Status für gleichgeschlechtliche Paare gesorgt, ${ }^{98}$ zugleich aber den in einzelnen Vertragsstaaten der Europäischen Menschenrechtskonvention (EMRK) bestehenden besonderen Sensibilitäten hinsichtlich des Status' der Ehe Rechnung getragen. ${ }^{99}$ Aufgrund dieser Rechtsprechung müssen die Vertragsstaaten die gleichgeschlechtlichen Beziehungen mindestens als eingetragene Partnerschaft anerkennen, sind aber nicht verpflichtet, diese als Ehe zu bezeichnen. ${ }^{100}$

95 EGMR, Vajnai gegen Hungary, App. No. 33629/06, Urteil v. 8.7.2008, Rn. 57.

96 Protokoll (Nr. 30) über die Anwendung der Charta der Grundrechte auf Polen und das Vereinigte Königreich, ABl. 115 v. 9.5.2008, 313-314.

97 Obergefell v. Hodges, 576 U.S. _ (2015).

98 EGMR, Vallianatos and Others v. Greece, App. Nos. 29381/09 and 32684/09, Urteil v. 7.11.2013.

99 EGMR, Schalk and Kopf v. Austria, App. No. 30141/04, Urteil v. 24.6.2010; EGMR, Hämäläinen v. Finland, App. No. 37359/09, Urteil v. 16.7.2014; EGMR, Chapin und Charpentier v. Frankreich, App. No. 40183/07, Urteil v. 9.6.2016.

100 EGMR, Schalk and Kopf (Fn. 99), Rn. 62. 


\section{Die dogmatische Ebene: die unmittelbare Wirkung von Artikel 2 EUV}

Die nächste Frage ist, ob und wie die oben dargelegte Lehre ihren Weg in das positive Recht finden kann. Leider bietet die herrschende Lehre hierfür nur einen geringen Spielraum. Es ist allgemein anerkannt, dass die Mitgliedstaaten, trotz der verschiedenen (bedeutenden, aber vereinzelten) Anforderungen, welche das EU-Recht hinsichtlich der Rechtsstaatlichkeit an die Wahrnehmung der eigenen Angelegenheiten durch die Mitgliedstaaten stellt, keinem umfassenden Regime unterworfen sind. Obwohl der EuGH die „Durchführung des EU-Rechts" weit auslegt, macht der Wortlaut von Artikel 51 der Charta es unmöglich, den Geltungsbereich der Charta auf alle Fälle auszuweiten, welche die europäischen Grundwerte berühren. EU-Recht enthält jedoch einen geeigneten Ausgangspunkt für die wichtigsten europäischen Rechtsstaatlichkeitsanforderungen: Artikel $2 \mathrm{EUV}$, der wegen seines allgemeinen Wortlauts durchaus übersehen wird. ${ }^{101}$ Artikel $2 \mathrm{EUV}$ ist ein adäquater Ausgangspunkt, weil er unmittelbare Wirkung hat und die diagonal anwendbaren europäischen Rechtsstaatlichkeitsanforderungen darin inkorporiert werden können.

Die Fragen, ob Artikel 2 EUV klar genug ist, um über unmittelbare Wirkung zu verfügen, und ob er als Anknüpfungspunkt für die europäischen Rechtsstaatlichkeitsanforderungen fungieren kann, sind miteinander verbunden. Der Text von Artikel 2 EUV ist vage formuliert, enthält aber bestimmte Begriffe, die im Lichte des europarechtlichen Rahmens gerichtlich ausgelegt werden können.

Die unmittelbare Wirkung ist eines der Grundprinzipien des EU-Rechts, das (zusammen mit dem Prinzip des Vorrangs) ${ }^{102}$ sicherstellt, dass die EUNormen ohne Weiteres vor nationalen Gerichten und Behörden geltend gemacht werden können. Obwohl die unmittelbare Wirkung nie für alle Bestimmungen des EU-Rechts gegolten hat und von Anfang an ausschließlich bei Vorliegen bestimmter Voraussetzungen zum Tragen gekommen ist, sind diese Bedingungen Schritt für Schritt in eine allgemeine Anerkennung der unmittelbaren Anwendbarkeit vor Gericht umgewandelt worden: Alle EU-Rechtsnormen, die einer gerichtlichen Auslegung zugänglich sind, verfügen über unmittelbare Wirkung. ${ }^{103}$

101 Für eine Ausnahme, siehe Halmai (Fn. 26), 487.

102 Siehe Amedeo Arena, The Twin Doctrines of Primacy and Pre-Emption, in: Robert Schütze/Takis Tridimas (Hrsg.), Oxford Principles of European Union Law: the European Union Legal Order, Vol. I, Oxford: Oxford University Press 2018, 300-349.

103 Bruno de Witte, Direct Effect, Primacy, and the Nature of the Legal Order, in: Paul P. Craig/Gráinne de Búrca (Hrsg.). The Evolution of EU Law, 2. Aufl., Oxford: Oxford University Press 2011, 323-362 (331). 
Ursprünglich galt die unmittelbare Wirkung für einen beschränkten Kreis von Normen. Im Fall Van Gend en Loos hat der EuGH einige Voraussetzungen für die unmittelbare Wirkung festgelegt: Die Norm muss klar, inhaltlich zwingend und unabhängig von einem internen Rechtsakt wirksam sein, sowie eine negative Verpflichtung enthalten. ${ }^{104}$ Später jedoch schwächte der EuGH diese Voraussetzungen erheblich ab.

Die Anforderung einer negativen Verpflichtung verlor ihre Bedeutung, nachdem der EuGH entschied, dass die Bürger sich auf Vorschriften von Richtlinien, die prozessuale Pflicht festlegen (z. B. Umweltverträglichkeitsprüfung), berufen können, um die Gültigkeit nationaler Maßnahmen anzufechten. ${ }^{105}$

„[I]n den Fällen, in denen die Gemeinschaftsorgane die Mitgliedstaaten durch eine Richtlinie zu einem bestimmten Verhalten verpflichten, würde deren praktische Wirksamkeit abgeschwächt, wenn die Bürger sich vor Gericht nicht auf sie berufen und die nationalen Gerichte sie nicht als Bestandteil des Gemeinschaftsrechts berücksichtigen könnten, um zu prüfen, ob der nationale Gesetzgeber im Rahmen der ihm vorbehaltenen Befugnis, Form und Mittel für die Umsetzung der Richtlinie zu bestimmen, innerhalb der von der Richtlinie gezogenen Grenzen seines Entscheidungsspielraums geblieben ist. " 106

Ursprünglich betonte die Rechtsprechung, dass solche Normen unmittelbare Wirkung haben können, die den Bürgern Rechte einräumen, und das Ziel der unmittelbaren Wirkung ist, sicherzustellen, dass mitgliedstaatliche Behörden und Gerichte diese Rechte auch durchsetzen. Später verschwand aber dieser Gedanke und wurde durch das Konzept ersetzt, dass alle EUNormen, auf die sich die Bürger vor mitgliedstaatlichen Behörden und Gerichten berufen können, unmittelbare Wirkung haben. ${ }^{107}$ Die Anforderung, dass die Norm klar und inhaltlich zwingend sein muss, wurde immer großzügiger interpretiert. Es war danach klar, dass die unmittelbare Wirkung nicht auf eindeutige Normen beschränkt ist. Eine solch enge Lesart würde nämlich die Mehrheit der EU-Normen ausschließen. Eine Norm kann ausreichend klar sein, auch wenn sie eine gerichtliche Auslegung erfordert.

Selbst wenn der Gesetzgeber den Auslegungsspielraum mit Absicht in die Norm „einbaut“, um den Mitgliedstaaten einen Ermessensspielraum ein-

104 EuGH, Urteil v. 5.2.1963, Rs. 26/62, Slg. 1963, 1, ECLI:EU:C:1963:1 - NV Algemene Transport- en Expeditie Onderneming van Gend E Loos gegen Netherlands Inland Revenue Administration.

105 De Witte (Fn. 103), 330-331.

106 EuGH, Urteil v. 19.9.2000, Rs. C-287/98, Slg. 2000, I-6917, ECLI:EU:C:2000:468 Rn. 32 - Luxembourg v. Berthe Linster.

107 De Witte (Fn. 103), 330. 
zuräumen, gibt es keinen Grund, nationale Maßnahmen einer - diesen Ermessensspielraum berücksichtigenden - rechtlichen Überprüfung nicht zu unterzuwerfen. ${ }^{108}$

Die Mitgliedstaaten haben einen weiten Ermessensspielraum, wenn sie den Handel zwischen den Mitgliedstaaten mit Verweis auf das öffentliche Interesse beschränken, ${ }^{109}$ trotzdem haben die Binnenmarktregeln eine unmittelbare Wirkung. Der EuGH hat festgestellt, dass der Umstand, dass eine Norm den Mitgliedstaaten Wahlfreiheit einräumt, nicht bedeutet, dass sie keine unmittelbare Wirkung hätte.

„Wie sich jedoch schon implizit aus der Rechtsprechung des Gerichtshofes [...] ergibt, schließt die Tatsache, daß der Staat zwischen mehreren möglichen Mitteln zur Erreichung des durch eine Richtlinie vorgeschriebenen Ziels wählen kann, nicht aus, daß der einzelne vor den nationalen Gerichten die Rechte geltend machen kann, deren Inhalt sich bereits aufgrund der Richtlinie mit hinreichender Genauigkeit bestimmen läßt."

Diese Entwicklung bedeutet, dass die Voraussetzungen der unmittelbaren Wirkung auf eine Schlüsselfrage reduziert werden können: wenn eine Norm gerichtlich ausgelegt werden kann, verfügt sie über unmittelbare Wirkung. ${ }^{111}$ Generalanwalt van Gerven fasste das Ergebnis dieser Rechtsentwicklung in seinem Schlussantrag im Fall British Coal Corporation eindeutig zusammen.

„Sofern und soweit eine Gemeinschaftsbestimmung für sich allein hinreichend brauchbar für die Anwendung durch das Gericht ist, entfaltet sie unmittelbare Wirkung. Die Deutlichkeit, Genauigkeit, Unbedingtheit, Vollständigkeit oder Perfektion der Norm und der Gesichtspunkt, daß diese keiner im Ermessen stehenden Durchführungsmaßnahme bedarf, sind in dieser Hinsicht nur Facetten ein und desselben Merkmals, das die Regelung aufweisen muß, nämlich, daß sie geeignet sein muß, vom Gericht auf einen Einzelfall angewandt zu werden.“112

108 Siehe de Witte (Fn. 103), 330.

109 Artikel 36 AEUV.

110 EuGH, Urteil v. 19.11.1991, verb. Rs. C-6/90 und C-9/90, Slg. 1991, I-05357, ECLI:EU: C:1991:428, Rn. 17 - Andrea Francovich und Danila Bonifaci gegen Italien. Siehe EuGH, Urteil v. 2.8.1993, Rs. C-271/91, Slg. 1993, I-04367, ECLI:EU:C:1993:335, Rn. 37 - M. Helen Marshall gegen Southampton and South-West Hampshire Area Health Authority.

111 De Witte (Fn. 103), 330. Siehe Pierre Pescatore, The Doctrine of Direct Effect: An Infant Disease of Community Law, EL Rev. 8 (1983), 155-177 (177); Sacha Prechal, Direct Effect Reconsidered, Redefined and Rejected, in: Jolande M. Prinssen/Annette Schrauwen (Hrsg.), Direct Effect - Rethinking a Classic of EC Legal Doctrine, Groningen: Europa Law Publishing 2002, 15-42 (22).

112 Schlussanträge des Generalanwalts Van Gerven in EuGH, Urteil v. 13.4.1994, Rs. C128/92, Slg. 1994, I-01209, ECLI:EU:C:1993:860 - H. J. Banks \& Co. Ltd gegen British Coal Corporation. 
Das wichtigste Argument gegen die gerichtliche Auslegungsfähigkeit (Anwendbarkeit) von Artikel 2 EUV ist, dass sein Wortlaut generell ist und er eher eine politische Grundsatzerklärung ohne echten normativen Inhalt ist. ${ }^{113}$ Dieses Argument lässt jedoch außer Betracht, dass das Ziel von Artikel 2 EUV ist, für die Mitgliedstaaten eine Verpflichtung aufzustellen ${ }^{114}$ (sonst wäre diese Vorschrift in der Präambel gesetzt worden) und der Inhalt dieser Verpflichtung gerichtlich bestimmt werden kann, da die in dieser Vorschrift verwendeten Begriffe einen allgemein anerkannten Inhalt haben. Wenn Artikel 2 EUV den traditionellen Methoden der Gesetzesauslegung, besonders der systematischen Auslegung, unterworfen wird, stellt sich heraus, dass der Inhalt dieser Vorschrift schlüssig zu bestimmen ist. Obwohl sein Wortlaut in der Tat generell ist und eine grammatikalische Auslegung an sich schwierig macht, sind die hier aufgezählten Begriffe anhand der europarechtlichen Dogmatik auslegungsfähig. Das dogmatische System des EU-Rechts füllt die im Artikel 2 EUV verwendeten Begriffe mit Inhalt. Dieser Ansatz, d.h., dass die Begriffe einer Norm anhand der Bestimmungen anderer Normen ausgelegt werden können, ist weder neu noch unbekannt. Für den Fall, dass die grammatikalische Auslegung einer Bestimmung zu keinem klaren Ergebnis führt, ist allgemein anerkannt, dass sowohl die anderen Normen als auch Absicht des Gesetzgebers als Auslegungshilfe herangezogen werden können. Die Richter können auf diese Weise selbst sehr allgemein und unbestimmt formulierte Vorschriften auslegen, wenn diese in anderen Vorschriften näher bestimmte Begriffe verwenden.

Artikel 2 EUV enthält ohne Zweifel Begriffe (z. B. Rechtsstaatlichkeit, Menschenrechte), die aus sich heraus keinen eindeutigen rechtlichen Inhalt ergeben. Wenn man aber Artikel 2 EUV einer systematischen Auslegung unterwirft, kommt man zu dem Schluss, dass diese Begriffe im EU-Recht einen relativ gut zu bestimmenden Inhalt haben. Das EU-Recht ist ein kohärentes System und die Einzelnormen müssen im Lichte des generellen Systems des EU-Rechts ausgelegt werden. Es ist bemerkenswert, dass der EuGH keinen Skrupel hatte, einen normativen Grund in die allgemeinen Rechtsgrundsätze hineinzulesen, obwohl dies im EU-Recht nicht vorgesehen war.

Dieser Ansatz ist im Verfassungsrecht nicht unbekannt. In der Tat lassen sich in den nationalen Verfassungsordnungen zahlreiche Beispielsfälle finden,

113 Die Vorschriften des EUV haben denselben rechtlichen Status wie die des AEUV, daher sind deren unmittelbare Wirkung gemäß denselben Grundsätzen zu beurteilen. Das wird durch Artikel 1 EUV und Artikel 1 Absatz 2 AEUV klargestellt: beide sehen vor, dass „beide Verträge $[\ldots]$ rechtlich gleichrangig" sind.

114 Siehe Closa/Kochenov/Weiler (Fn. 9), 10 ("Article 7 TEU may be read in a way that indicates that Article 2 TEU imposes an obligation on the Member States.”). 
in denen die Gerichte schwer bestimmbare Begriffe mit Inhalt gefüllt haben. So erklärt das Grundgesetz die Menschenwürde für unantastbar, ${ }^{115}$ ohne diesen Begriff zu definieren. Eine ähnliche Vorschrift findet man in der spanischen Verfassung. ${ }^{116}$ Die französische Erklärung der Menschen- und Bürgerrechte von 1789 (Déclaration des Droits de l'Homme et du Citoyen) verkündet, dass „die Menschen frei und gleich an Rechten geboren werden und bleiben", ohne die Bedeutung dieser Freiheit zu bestimmen. Das ungarische Grundgesetz bestimmt, dass Ungarn ein Rechtsstaat ist, ${ }^{117}$ aber es gibt keine Definition oder konkrete Anhaltspunkte für das Verständnis dieses Begriffs. Wenn Artikel 2 EUV in diesen Kontext gesetzt wird, stellt sich heraus, dass sein Inhalt ohne Weiteres gerichtlich zu bestimmen ist. Die relevante Frage ist nicht, ob der Text von Artikel 2 EUV an sich klar und eindeutig ist, sondern ob ihm, anhand des EU-Rechts, eine ausreichend klare Bedeutung zugeschrieben werden kann.

Aus dieser Perspektive ist es unerheblich, ob Artikel 2 EUV den Inhalt der verwendeten Begriffe bestimmt. Die relevante Frage ist, ob diese Begriffe im Lichte anderer Regeln des EU-Rechts ausreichend klar bestimmt werden können, sodass die Gerichte sie auslegen und anwenden können. Artikel 2 EUV ist, in der Tat, ein „verfassungsrechtlicher Blankett-Tatbestand". Ähnlich einem offenen Tatbestand im Strafrecht, der durch Inkorporation von äußeren Regeln und Begriffen vervollständigt wird, kann Artikel 2 EUV als Ausgangspunkt für die europäischen Rechtsstaatlichkeitsanforderungen dienen. Wenn solch eine Konstruktion mit den sehr strengen Grundsätzen des Strafrechts vereinbar ist, sollte sie in anderen Rechtsgebieten a fortiori akzeptabel sein.

Das EU-Recht enthält ein detailliertes System von Rechtsstaatlichkeitsanforderungen, die von Anfang an Teil des EU-Rechts waren. Der EuGH hat die Rechtsgrundsätze als primäre Quellen des EU-Rechts angesehen und hat das Prinzip der Rechtsstaatlichkeit zum Bestandteil des EU-Rechts gemacht. Mit der umfassenden Kodifikation der europäischen Grundrechte in der Charta der Grundrechte der Europäischen Union sind diese Standards viel klarer geworden. Der Geltungsbereich der Charta ist beschränkt (sie ist auf die Mitgliedstaaten nur bei Durchsetzung des EU-Rechts anwendbar). Unabhängig davon, enthält die Charta eine klare und vollständige Liste der von der EU anerkannten Grundrechte und bestimmt dadurch, was im EU-Recht unter „Menschenrechte“ verstanden wird.

115 Artikel 1(1) Grundgesetz.

116 Artikel 10(1) der Verfassung von Spanien.

117 Artikel B) des ungarischen Grundgesetzes. 
Die vorgeschlagene Auslegung von Artikel 2 EUV ist nicht ohne Präzedenzfall. In der Tat hat der EuGH das Rechtsstaatlichkeitsprinzip zwei Mal als Bestandteil des Gemeinschaftsrechts anerkannt und für unmittelbar wirksam erklärt. In beiden Fällen hatte die Inkorporation einen schwächeren normativen Grund als Artikel 2 EUV.

Erstens, im Fall Wachauf entschied der EuGH dass die Rechtsgrundsätze bei der Durchführung des Gemeinschaftsrechts auch auf die Mitgliedstaaten anwendbar sind. ${ }^{118}$ Diese Rechtsprechung ist durch Artikel 51 der Charta kodifiziert worden. Der EuGH hat die Pflicht, die Grundrechte zu achten, ohne „textliche“ Grundlage formuliert und er hat nicht einmal untersucht, ob die Voraussetzungen einer unmittelbaren Wirkung erfüllt waren. Dagegen nimmt Artikel 2 EUV, der kein Richterrecht ist, ausdrücklich auf das Rechtsstaatlichkeitsprinzip Bezug. Wenn aber Inkorporation und die unmittelbare Wirkung des Prinzips im Fall Wachauf in gültiger Weise vom EuGH deduziert werden konnten, sollten sie auch in Bezug auf Artikel 2 EUV keine unüberwindlichen juristischen Probleme aufwerfen.

Im Fall ERT $T^{119}$ erklärte der EuGH zudem das europäische Rechtsstaatlichkeitsprinzip auf die Mitgliedstaaten in einer der diagonalen Anwendung sehr ähnlichen Lage für anwendbar. Im Binnenmarktrecht können danach die Mitgliedstaaten die Beschränkung des freien Verkehrs von Personen, Waren, Dienstleistungen und Kapital mit Bezugnahme auf das örtliche öffentliche Interesse rechtfertigen, aber nur dann, wenn die Maßnahme mit dem europäischen Rechtsstaatlichkeitsprinzip vereinbar ist. Der EuGH las also mit anderen Worten das Rechtsstaatlichkeitsprinzip in die verschiedenen Ausnahmen von den Grundfreiheiten hinein. Es ist bemerkenswert, dass in diesem Fall der EuGH das Rechtsstaatlichkeitsprinzip auf die Mitgliedstaaten ohne normative Basis angewendet hat. Im Gegensatz dazu enthält Artikel 2 EUV eine explizite Bezugnahme

\section{Die institutionelle Ebene: Legalisierung und Entpolitisierung}

In der derzeitigen institutionellen Architektur der EU haben die politischen Elemente eine zu starke Rolle, während die gerichtliche Kontrolle nur eine untergeordnete Bedeutung hat. Der einzig umfassende und unmittelbare

118 EuGH, Urteil v. 13.7.1989, Rs. 5/88, Slg. 1989, 2609, ECLI:EU:C:1989:321, Rn. 19 Wachauf.

119 EuGH, ERT (Fn. 21). Siehe Robert Schütze, European Fundamental Rights and the Member States: From 'Selective' to 'Total' Incorporation?, Cambridge Yearbook of European Legal Studies 14 (2012), 337-361 (348-351). 
Mechanismus, der zur Verfügung steht, um Rechtsstaatlichkeitsprobleme in den Mitgliedstaaten zu beseitigen, ist Artikel 7 EUV: ein genuin politischer Mechanismus, der auf systematische Probleme und nicht individuelle Rechtsverletzungen abzielt.

Das EU-Recht enthält vereinzelte Vorschriften, die für den Schutz der Rechtsstaatlichkeit in den Mitgliedstaaten in Anspruch genommen werden können. Diese bieten aber keine umfassende Lösung. Die Praxis, dass die Kommission sich auf die "Nebenwirkungen“ sachlich nicht zusammenhängender Vorschriften beruft, ist bedenklich, da sie Probleme der Vorhersehbarkeit aufwirft und die Gefahr mit sich bringt, dass die Absicht, die Rechtsstaatlichkeit zu verteidigen, die objektive Auslegung dieser nicht zusammenhängenden Normen stört und zur Politisierung des Gerichtsverfahrens führt.

Es wäre sehr wichtig, diese Instrumente durch echte Justizmechanismen zu ergänzen, wie sie auch sonst der Durchsetzung des EU-Rechts zur Verfügung stehen. Diese gerichtliche Durchsetzung muss zwei Anforderungen erfüllen: sie muss sowohl individuelle als auch systematische Rechtsverletzungen erfassen und entpolitisiert sein. Das Vorabentscheidungsverfahren könnte in Fällen eingeleitet werden, in denen individuelle Abhilfe gesucht wird, während das Vertragsverletzungsverfahren für die Behebung systematischer Probleme genutzt werden könnte.

Die Doppelstruktur des vierzehnten Zusatzartikels der amerikanischen Verfassung bietet eine interessante Parallele. Die in Abschnitt 1 des Zusatzartikels normierte Due Process-Klausel enthält einige diagonal geltende Freiheitsrechte, die als Ausgangspunkt für die gerichtliche Inkorporation dienen und Gegenstand von Individualrechtsstreitigkeiten sein können. Darüber hinaus gibt Abschnitt 5 dem Kongress eine positive und gerichtlich kontrollierte Gesetzgebungsbefugnis zur Durchsetzung dieser Grundrechte. Die Kombination des Vorabentscheidungs- mit dem Vertragsverletzungsverfahren würde diese Doppelstruktur im EU-Recht widerspiegeln.

\section{Fazit}

Die diagonale Anwendung des Rechtsstaatlichkeitsprinzips in der EU ist eine der Kernfragen der europäischen Integration geworden, die auf eine für die EU gefährliche Entwicklung reagiert. Während der horizontale Schutz der europäischen Grundwerte (d.h. deren Durchsetzung gegen die EU-Organe und die im Auftrag der EU handelnden Mitgliedstaaten) robust und umfassend ist, ist der diagonale Schutz dieser Grundwerte (d.h. die Durchsetzung gegen die in ihren eigenen Angelegenheiten handelnden Mitglied- 
staaten) defizitär. Es ist unbestritten, dass die derzeitige Architektur des EURechts keinen ausreichenden Schutz der Rechtsstaatlichkeit in den Mitgliedstaaten vorsieht.

Die vergleichende Föderalismusforschung bietet verschiedene Modelle und Ansätze, die zum besseren Verständnis der Diagonalitätsfrage beitragen können. In Kanada gilt die Bundescharta der Grundrechte gleichermaßen für die Bundesregierung wie für die Provinzen, letztere verfügen jedoch über ein Derogationsrecht und in der Rechtsprechung wird dem Föderalismusgedanken umfassend Rechnung getragen. In Australien enthält die Bundesverfassung keinen Grundrechtskatalog. Die Inkorporationsdoktrin des amerikanischen Verfassungsrechts bietet einen sehr lehrreichen Bezugspunkt und ein Modell, das als Inspirationsquelle und ein mutatis mutandis anwendbares Muster für Europa dienen kann.

Das EU-Recht braucht eine „kopernikanische Wende“, um seine Identität und Effektivität zu bewahren. Diese sollte darin bestehen, das Paradigma des Anwendungsbereichs, bei dem der Anwendungsbereich des EU-Rechts im Zentrum des europäischen Rechtsstaatlichkeitsuniversums steht, durch das Paradigma des Kernbereichsschutzes der Grundwerte zu ersetzen. Anstatt den Anwendungsbereich des EU-Rechts zu überdehnen, sollte das EU-Recht die Handlungen der Mitgliedstaaten in den Mittelpunkt der Betrachtungen stellen. In diesem Modell wird das europäische Rechtsstaatlichkeitsprinzip nicht vertikal, sondern diagonal angewendet: Es wirkt nicht in vollem Umfang, sondern nur insoweit es zur Wahrung der Grundwerte erforderlich ist.

Die diagonal anwendbaren Rechtsstaatlichkeitsanforderungen sollten über Artikel 2 EUV zur Geltung gebracht werden. Damit könnte ihre Verletzung sowohl in Vertragsverletzungs-, als auch in Vorabentscheidungsverfahren vor Gericht gerügt werden. Die vorgeschlagene selektive Inkorporation wird durch ontologische, dogmatische und institutionelle Erwägungen gerechtfertigt.

Die diagonale Anwendung des europäischen Rechtsstaatlichkeitsprinzips ist durch zwei Umstände gerechtfertigt. Der prinzipielle Grund ist, dass die Verletzung der Kernwerte ein „Scheidungsgrund“ ist, weil sie mit der europäischen Identität unvereinbar ist. Der praktische Grund ist, dass die auf dem gegenseitigen Vertrauen zwischen den Mitgliedstaaten beruhende Arbeitsweise der EU (wie die Zusammenarbeit und gegenseitige Anerkennung im Bereich der Zivil- und Strafjustiz oder die dezentrale Durchsetzung der EUWettbewerbsregeln) nur dann bewahrt werden kann, wenn das „europäische Wertminimum“ in allen Mitgliedstaaten eingehalten wird.

Diese ontologischen Überlegungen prägen den diagonalen Anwendungsbereich des europäischen Rechtsstaatlichkeitsprinzips: Anders als die vollständige und umfassende horizontale Anwendung beschränkt sich die dia- 
gonale Anwendung auf die Durchführung eines „europäischen Werteminimums“, das als Grundlage der Rechtstaatlichkeit auf EU-Ebene dient. Diese Ontologie impliziert, dass nicht alle in der Charta aufgelisteten Grundrechte diagonal anzuwenden sind (selektive Inkorporation), und auch diejenigen, die danach für die Mitgliedstaaten bei der Wahrnehmung ihrer eigenen Angelegenheiten gelten, unter Berücksichtigung des Ermessensspielraums der Mitgliedstaaten angewendet werden müssen.

Artikel 2 EUV dient als geeigneter dogmatischer Ausgangspunkt für die vorgeschlagene Diagonalitätslehre und für die Durchsetzung des europäischen Werteminimums. Obwohl der Wortlaut von Artikel 2 EUV allgemein gehalten ist, nimmt er auf Begriffe Bezug (Rechtsstaatlichkeit, Menschenrechte), die im EU-Recht hinreichend geklärt sind und daher die Voraussetzungen der unmittelbaren Wirkung erfüllen. Es gibt zwei Grunderwägungen, welche die Auslegung von Artikel 2 EUV bestimmen müssen, und der „Kernwerte“-Ansatz würde beiden entsprechen. Einerseits verlangt der Grundsatz der begrenzten Einzelermächtigung (der den berechtigten Erwartungen der Mitgliedstaaten entspricht), dass das europäische Rechtsstaatlichkeitsprinzip nicht in vollem Umfang auf die Mitgliedstaaten angewendet wird, weil die letzteren auf die Union nie eine umfassende Zuständigkeit zum Schutz der Menschenrechte übertragen haben. Andererseits haben die Mitgliedstaaten mit der Annahme von Artikel 2 EUV ausdrücklich die Verpflichtung akzeptiert, das europäische Rechtsstaatlichkeitsprinzip zu beachten, daher können die aus dieser Vorschrift entspringenden Anforderungen nicht ihren berechtigten Erwartungen widersprechen.

\section{Summary: A Proposal for a Solution to the European Rule of Law Crisis: the Doctrine of Diagonality}

The article makes a proposal for the solution of the European rule-of-law problem. It demonstrates that EU law should follow the doctrine of diagonality and the currently prevailing paradigm of scope should be replaced with the paradigm of core values. The proposal makes use of the comparative law of multi-layered constitutional systems, in particular the doctrine of incorporation of US constitutional law. In the first step, the article takes stock of the proposals made in the scholarship and explains why these have failed to solve the problem. In the second step, it presents the new concept of diagonality to describe the European rule-of-law crisis, provides on overview of the (missing) doctrine of diagonality and the criticizes the prevailing paradigm of scope. In the third step, the article presents that diagonality is a typical 
phenomenon of multi-layered systems and comparative law can contribute to the solution of the rule-of-law problem. Finally, the article presents the doctrine of diagonality and explains why the prevailing paradigm of scope should be replaced with the paradigm of core values. It demonstrates that Article 2 TEU is an adequate entry point for the application of the doctrine of diagonality and the CJEU should enforce the European rule-of-law requirements against the Member States via Article 2 TEU.

\section{Keywords}

Rule of Law - Constitutional Backsliding - Constitutional Identities Multi-Layered Constitutional Systems 
